Archive for

Organic Chemistry
Arkivoc 2018, part vii, 0-0

to be inserted by editorial office

\title{
Synthesis and insecticidal activity of novel benzothiazole derivatives containing the coumarin moiety
}

\author{
Wei-Jie Si, ${ }^{a, b, c}$ Min Chen, ${ }^{a, b}$ Xue-Lun Wang, ${ }^{a, b}$ Meng-Qi Wang, ${ }^{\text {a,b Jian Jiao, }}$, ${ }^{a, b}$ \\ Xin-Can Fu, ${ }^{a, b}$ and Chun-Long Yang*a,b,c
}

${ }^{a}$ Department of Chemistry, College of Sciences, Nanjing Agricultural University, Nanjing 210 095, China

${ }^{b}$ Jiangsu Key Laboratory of Pesticide Science, Nanjing Agricultural University, Nanjing 210 095, China

${ }^{c}$ Key Laboratory of Monitoring and Management of Crop Diseases and Insect Pests, Ministry of Agriculture, Nanjing Agricultural University, Nanjing 210 095, China

Email:ycl@njau.edu.cn

Received 06-19-2018

Accepted 08-06-2018

Published on line 09-06-2018

\section{Abstract}

A series of novel benzothiazole derivatives containing a coumarin moiety was designed and synthesized. Their structures were characterized in detail via ${ }^{1} \mathrm{H} N M R,{ }^{13} \mathrm{C} N M R$, HRMS spectra and single-crystal X-ray diffraction analysis. The bioassay results indicated that some compounds showed encouraging insecticidal activity against Plutella xylostella and Aphis fabae at $400 \mathrm{mg} / \mathrm{L}$. The preliminary structure-activity relationships were also discussed.

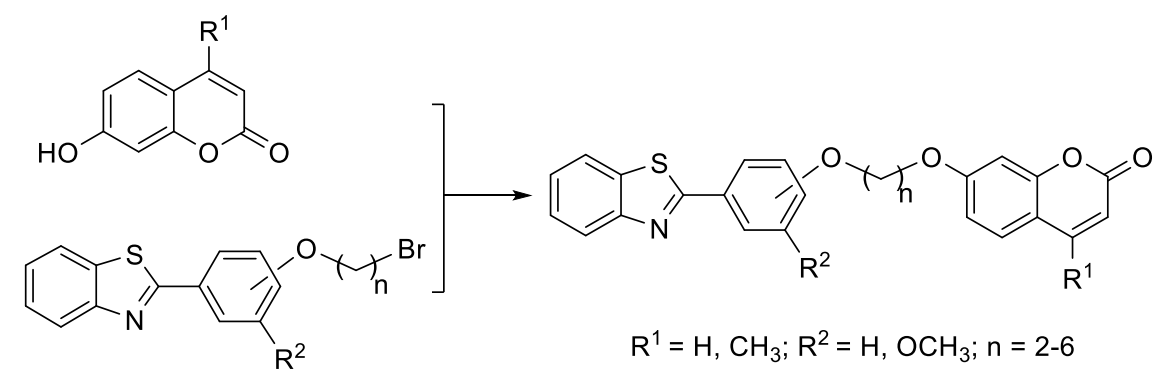

Keywords: Synthesis, benzothiazole, coumarin, insecticidal activity, structure-activity relationship 
The growth, transportation and storage of crops are endangered by various agricultural diseases, insects, grasses, and rats. These agricultural harmful organisms have seriously affected the growth of crops, the appearance, yield and quality of agricultural products. Because agricultural pests are characterized by many types, large quantities, and serious damage, it is an important task in agricultural production to control agricultural pests and reduce their damage. ${ }^{1}$ Pesticides have played an important role in the prevention and control of agricultural pests. However, drug resistances, environmental hazards and other problems have become increasingly prominent with the use of insecticides, ${ }^{2,3}$ which objectively require the discovery of new insecticide candidates.

Benzothiazole derivatives exhibit a wide variety of biological activities such as anticancer, ${ }^{4}$ analgesic, ${ }^{5}$ antiinflammatory, ${ }^{6}$ anti-tubercular, ${ }^{7}$ antimicrobial, ${ }^{8}$ and insecticidal activity. ${ }^{9}$ The compounds A and B (Figure 1) containing benzothiazole moiety possessed insecticidal activity against Prodenia litura and Plutella xylostella, respectively. ${ }^{10,11}$ The compound $C$ containing phenyl benzothiazole moiety showed acaricidal activity. ${ }^{12}$ Coumarins are frequently used to develop novel lead compounds for agrochemical and pharmaceutical because of their wide spectrum of bioactivities. ${ }^{13}$ Coumarin derivatives have often acted as anticancer prodrugs, ${ }^{14}$ or as antimalarial, ${ }^{15}$ anti-inflammatory, ${ }^{16}$ antioxidant and antimicrobial agents. ${ }^{17}$ In particular, compounds $D$ and $E$, which both contain coumarin skeleton in their structures have been reported to show insecticidal activity. ${ }^{18,19}$ However, almost no coumarin insecticides have actually been used to control agricultural insects. So the design and synthesis of the compounds containing the coumarin moiety is very meaningful for the development of novel insecticides with unique molecular skeleton.

In view of the above reports, two pharmacophores benzothiazole and coumarin were connected by an alkylene linker in the same molecule to design and synthesize a series of benzothiazole derivatives containing the coumarin group according to the "active substructure combination principle" (Figure 1). Subsequently, the insecticidal activity against Plutella xylostella and Aphis fabae (broad bean aphid) were evaluated. The preliminary structure-activity relationships between the title compounds and insecticidal activity were also analyzed.

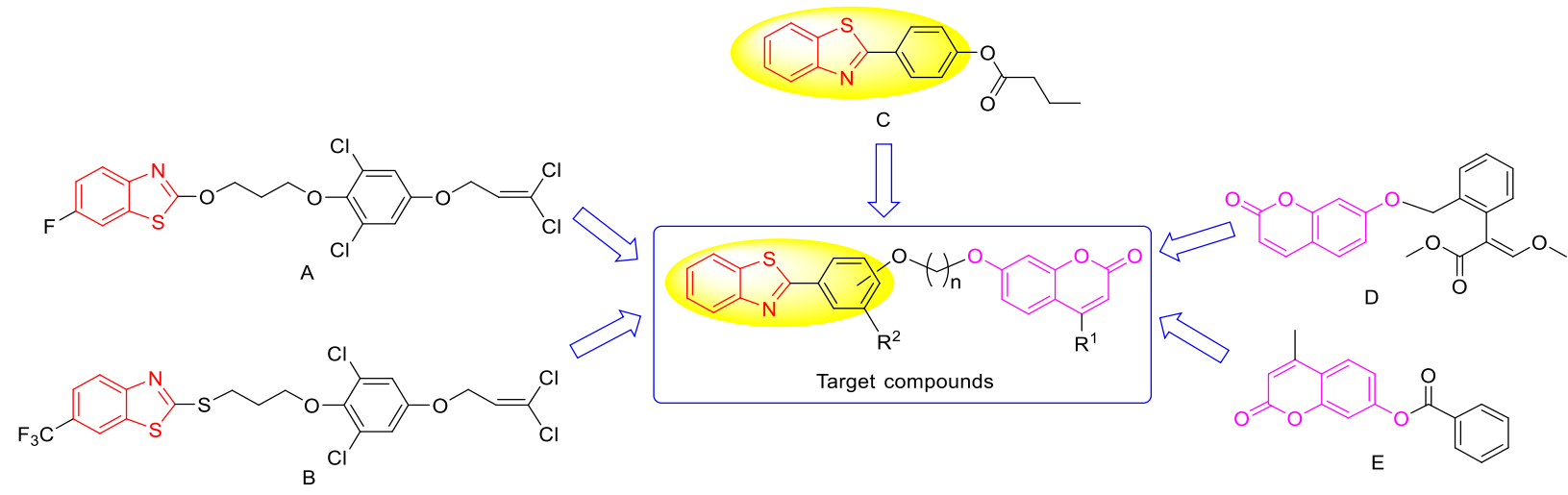

Figure 1. Design of target compounds. 


\section{Results and Discussion}

Chemistry. Schemes 1 and 2 describe the synthesis and structures of the intermediates and title compounds in the present study. Firstly, resorcinol 1 was reacted with 2-hydroxysuccinic acid and ethyl acetoacetate respectively to generate the corresponding coumarin intermediates 7-hydroxy-2H-chromen-2-one 2a and 7hydroxy-4-methyl-2H-chromen-2-one 2b. 2-Aminothiophenol was reacted respectively with 4hydroxybenzaldehyde, vanillin and 3-hydroxybenzaldehyde in the presence of sodium metabisulfite to obtain the benzothiazole intermediates $\mathbf{4 a - 4 c}$. Then the compounds $\mathbf{4}$ were treated respectively with dibromoalkanes $\left(\mathrm{Br}\left(\mathrm{CH}_{2}\right)_{n} \mathrm{Br}, \mathrm{n}=2-6\right)$ in the presence of $\mathrm{K}_{2} \mathrm{CO}_{3}$ to provide the brominated intermediates 5a-5o. Finally, the compounds $\mathbf{5}$ were reacted with compounds $\mathbf{2 a}$ and $\mathbf{2} \mathbf{b}$ respectively to get the target products $\mathbf{6 a -}$ $6 z$ and $6 a a-6 d d$ with yields ranging from $44.6 \%$ to $78.3 \%$.

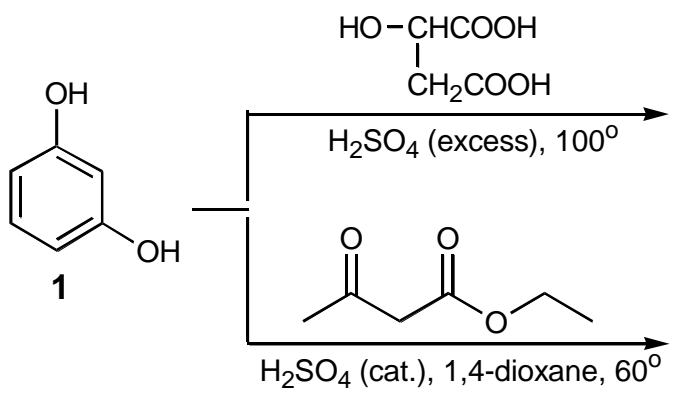<smiles>O=c1ccc2ccc(O)cc2o1</smiles>

2a<smiles>Cc1cc(=O)oc2cc(O)ccc12</smiles>

2b

Scheme 1. Synthesis of intermediate compounds $\mathbf{2 a}$ and $\mathbf{2 b}$.
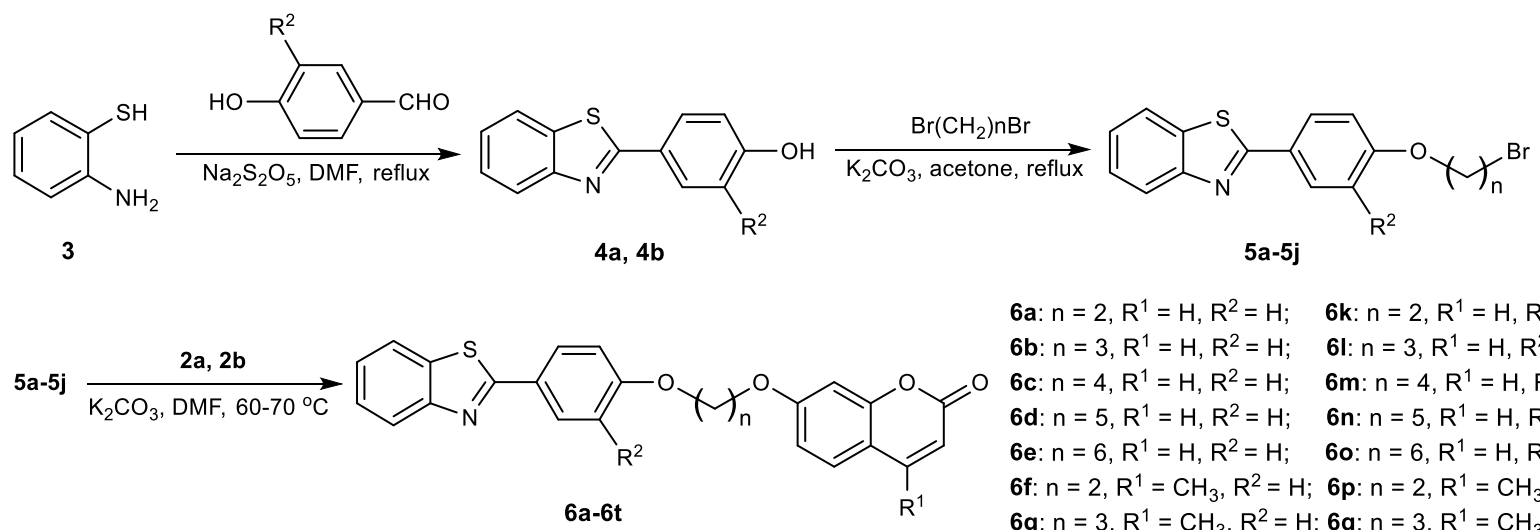

6a: $n=2, R^{1}=H, R^{2}=H ; \quad 6 k: n=2, R^{1}=H, R^{2}=\mathrm{OCH}_{3} ;$ 6b: $n=3, R^{1}=H, R^{2}=H ; \quad 6 I: n=3, R^{1}=H, R^{2}=O_{3}$; 6c: $n=4, R^{1}=H, R^{2}=H ; \quad 6 m: n=4, R^{1}=H, R^{2}=O^{2} H_{3}$; 6d: $n=5, R^{1}=H, R^{2}=H ; \quad 6 n: n=5, R^{1}=H, R^{2}=O^{2} H_{3}$; 6e: $n=6, R^{1}=H, R^{2}=H ; \quad 6 o: n=6, R^{1}=H, R^{2}=O_{3} H_{3}$; 6f: $n=2, R^{1}=C_{3}, R^{2}=H ; 6 p: n=2, R^{1}=C_{3}, R^{2}=O^{2} H_{3}$; 6g: $n=3, \mathrm{R}^{1}=\mathrm{CH}_{3}, \mathrm{R}^{2}=\mathrm{H} ; \mathbf{6 q}: \mathrm{n}=3, \mathrm{R}^{1}=\mathrm{CH}_{3}, \mathrm{R}^{2}=\mathrm{OCH}_{3}$; 6h: $n=4, R^{1}=C_{3}, R^{2}=H ; 6 r: n=4, R^{1}=C_{3}, R^{2}=O C_{3}$; 6i: $n=5, R^{1}=C_{3}, R^{2}=H ; 6 s: n=5, R^{1}=C_{3}, R^{2}=O^{2} H_{3}$;

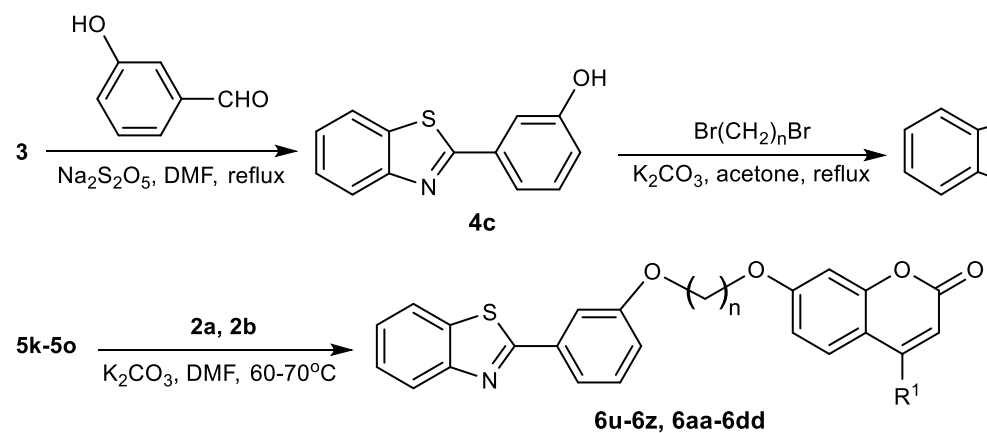
6j: $n=6, R^{1}=C_{3}, R^{2}=H$; 6 t: $n=6, R^{1}=C_{3}, R^{2}=O_{3}$.

Scheme 2. Synthesis route of title compounds $6 a-6 z$ and $6 a a-6 d d$. 
The ${ }^{1} \mathrm{H} N M R,{ }^{13} \mathrm{C} N M R$, and HRMS spectra data of the title compounds were fully in accordance with their assigned structures. The structures of some key intermediates were also confirmed by ${ }^{1} \mathrm{H} N M R$ spectra. The yields, melting points and spectra data of the title compounds and intermediates are listed in the Experimental Section. This is the first report of all of the title compounds.

Crystal structure of compound $\mathbf{6 v}$. The structure of compound $\mathbf{6 v}$ was further studied using the single crystal $\mathrm{X}$-ray analysis. The corresponding crystal structure and crystal packing diagrams are shown by Figure 2 . and Figure 3, respectively. As shown in Figure 2, the sulfur and nitrogen atoms of the benzothiazole ring appeared in a disordered state. Thus two corresponding intramolecular hydrogen bonds $\mathrm{C} 9-\mathrm{H} 9 \cdots \mathrm{S} 1{ }^{\prime}$ and $\mathrm{C} 13-\mathrm{H} 13 \cdots \mathrm{S} 1$ were formed. As shown in Figure 3, three intermolecular hydrogen bonds $\mathrm{C} 5-\mathrm{H} 5 \cdots \mathrm{S} 1, \mathrm{C} 12-\mathrm{H} 12 \cdots \mathrm{O} 1$ and $\mathrm{C} 23-$ $\mathrm{H} 23 \cdots \mathrm{O} 4$ were found. In addition, there were two offset face-to-face $\pi \cdots \pi$ stacking interactions between benzothiazole and chromene rings of two adjacent molecules. The intermolecular hydrogen bonds connected the molecules in the planes which were nearly perpendicular to the vector $b$. The $\pi \cdots \pi$ interactions made the molecules packed through the direction of vector $b$. These two actions made the molecules formed a 3-D supramolecular network structure. The crystal data for $6 v$ : triclinic, $a=6.5984(9) \AA, b=7.2742(12) \AA, c=$ 22.5363(19) $\AA, \alpha=90.238(10)^{\circ}, B=91.143(9)^{\circ}, \gamma=115.337(15)^{\circ}, V=977.4(3) \AA^{\circ}, Z=2$, space group P-1 (no.2), $\mu=0.201 \mathrm{~mm}^{-1}, D_{\text {calc }}=1.460 \mathrm{~g} / \mathrm{cm}^{3}, 7766$ reflections measured $(5.4 \leq 2 \theta \leq 52.8), 4000$ unique $\left(R_{\text {int }}=0.048\right)$ were used in all calculations. The final $R_{1}$ was $0.0806(\mathrm{I}>2 \sigma(\mathrm{I}))$ and $w R\left(\mathrm{~F}_{2}\right)$ was 0.2119 (all data). The crystallographic data have been deposited with the Cambridge Crystallographic Data Centre. The deposition number was CCDC1838383.

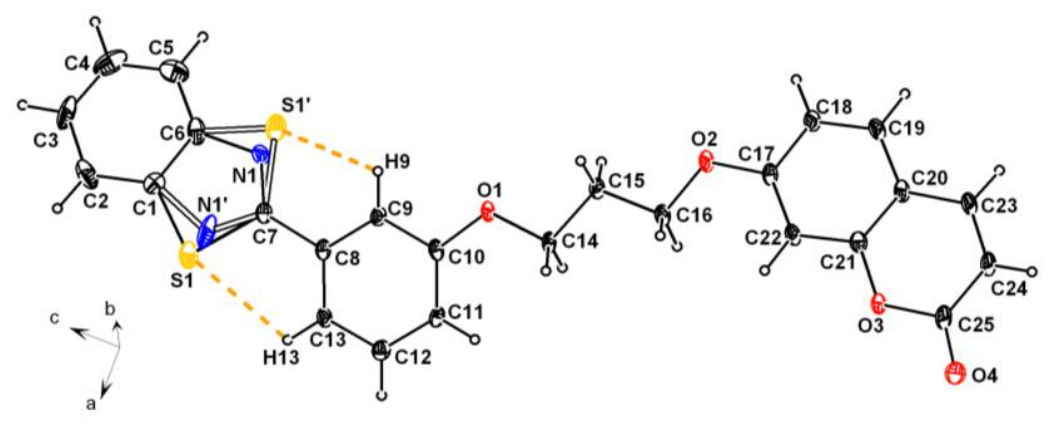

Yellow dashed lines show intramolecular hydrogen bonds.

Figure 2. Crystal structure diagram of compound $6 \mathbf{v}$.

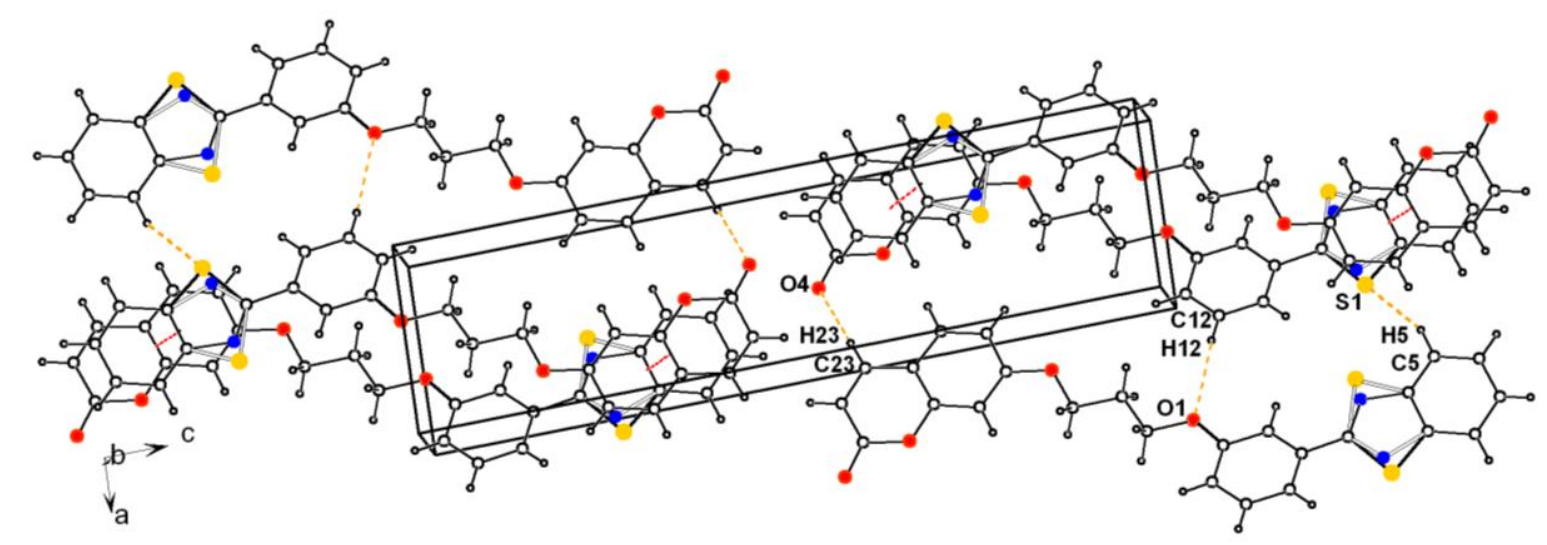

Yellow dashed lines show intermolecular hydrogen bonds. Red dashed lines show $\pi \cdots \pi$ interactions.

Figure 3. Crystal packing diagram of compound $6 \mathbf{v}$. 
Insecticidal activity. The results in Table 1 indicated that the title compounds showed moderate to significant insecticidal activity at $400 \mu \mathrm{g} / \mathrm{mL}$. Ten of the compounds showed high activity with the mortalities of more than $50 \%$ against $P$. xylostella. Among them, the compounds $\mathbf{6 d}, \mathbf{6 k}, \mathbf{6 w}$ and $\mathbf{6 z}$ possessed the mortalities of over $85 \%$, especially the compounds $6 \mathbf{d}$ and $6 \mathbf{w}$, which reached $100 \%$ and $93.10 \%$ mortalities, respectively. Meanwhile, twenty-three of the compounds showed high activity against the aphids, their mortalities were higher than $50 \%$. While the compounds $6 \mathrm{e}, 6 \mathrm{I}, 6 \mathrm{~s}, 6 \mathrm{t}$ and $6 \mathrm{u}$ presented noticeable activity with the mortalities of $90 \%$ above. Compared with the activity against $P$. xylostella, the title compounds showed higher insecticidal activity against $A$. fabae. Among them, the compounds $\mathbf{6 u}-\mathbf{6 z}$ and $\mathbf{6 a a}-\mathbf{6} \mathbf{d d}$ gave good activity against the aphids with the mortalities ranging from 56.67 to $96.51 \%$. That is to say, the alkylene linked at 3-position of benzothiazol-2-phenyl was beneficial to the bioactivity against the aphids. In addition, the compounds $6 \mathrm{c}, \mathbf{6 h}$, $6 \mathrm{~m}, 6 \mathrm{r}, \mathbf{6 w}$ and $\mathbf{6 b b}$ all exhibited evident activity against the aphids with the mortalities ranging from 60.00 to 88.41\%. These six compounds contain a same alkylene linker with four carbon atoms $(n=4)$.

Table 1. Insecticidal activity in vivo of title compounds at $400 \mu \mathrm{g} / \mathrm{mL}$

\begin{tabular}{|c|c|c|c|c|c|}
\hline \multirow{2}{*}{ Compd. } & \multicolumn{2}{|c|}{ Mortality (\%) } & \multirow{2}{*}{ Compd. } & \multicolumn{2}{|c|}{ Mortality (\%) } \\
\hline & $P . x^{a}$ & A. $f^{b}$ & & $P . x$. & A. $f$. \\
\hline $6 a$ & $32.26 \pm 3.23$ & $30.00 \pm 2.50$ & $6 q$ & $13.33 \pm 1.67$ & $16.22 \pm 2.70$ \\
\hline $6 b$ & $40.63 \pm 1.56$ & $80.57 \pm 3.43$ & $6 r$ & $48.28 \pm 3.45$ & $85.51 \pm 4.35$ \\
\hline $6 c$ & $43.75 \pm 4.69$ & $71.88 \pm 3.13$ & $6 s$ & $84.38 \pm 3.13$ & $96.47 \pm 3.53$ \\
\hline $6 d$ & 100 & $54.84 \pm 1.08$ & $6 t$ & $6.90 \pm 1.72$ & $96.51 \pm 3.49$ \\
\hline $6 e$ & $59.38 \pm 3.13$ & $92.13 \pm 2.25$ & $6 u$ & $13.33 \pm 1.67$ & $96.51 \pm 2.33$ \\
\hline $6 f$ & $50.00 \pm 5.00$ & $24.49 \pm 4.08$ & $6 v$ & $43.33 \pm 5.00$ & $71.72 \pm 3.03$ \\
\hline $6 g$ & $15.63 \pm 1.56$ & $22.58 \pm 2.15$ & $6 w$ & $93.10 \pm 3.45$ & $76.47 \pm 3.92$ \\
\hline $6 h$ & $48.48 \pm 4.55$ & $60.00 \pm 1.54$ & $6 x$ & $31.03 \pm 1.72$ & $56.67 \pm 4.44$ \\
\hline $6 i$ & $19.35 \pm 1.61$ & $60.98 \pm 4.88$ & $6 y$ & $26.67 \pm 3.33$ & $84.15 \pm 2.44$ \\
\hline $6 \mathbf{j}$ & $73.33 \pm 3.33$ & $26.09 \pm 4.35$ & $6 z$ & $86.21 \pm 6.90$ & $58.54 \pm 3.66$ \\
\hline $6 k$ & $86.67 \pm 1.67$ & $24.74 \pm 3.09$ & $6 a a$ & $63.33 \pm 6.67$ & $89.87 \pm 5.06$ \\
\hline 61 & $35.48 \pm 4.84$ & $94.34 \pm 4.72$ & $6 b b$ & $12.50 \pm 3.13$ & $88.41 \pm 4.35$ \\
\hline $6 m$ & $13.33 \pm 3.33$ & $87.72 \pm 5.26$ & $6 c c$ & $64.52 \pm 4.84$ & $68.42 \pm 3.51$ \\
\hline $6 n$ & $26.67 \pm 3.33$ & $27.94 \pm 2.94$ & $6 d d$ & $38.71 \pm 6.45$ & $86.76 \pm 4.41$ \\
\hline 60 & $3.33 \pm 1.67$ & $53.62 \pm 4.35$ & $\mathrm{Ch} .{ }^{\mathrm{c}}$ & 100 & 100 \\
\hline $6 p$ & $10.00 \pm 1.67$ & $74.58 \pm 3.39$ & $\mathrm{Im} .^{d}$ & 100 & 100 \\
\hline
\end{tabular}

a P.x., Plutella xylostella; ${ }^{\mathrm{b}}$ A.f., Aphis fabae; ${ }^{\mathrm{c}}$ Ch., chlorantraniliprole; ${ }^{\mathrm{d}} \mathrm{Im} .$, imidacloprid.

\section{Conclusions}

In summary, a series of novel benzothiazole derivatives containing a coumarin group were designed, prepared and evaluated for their insecticidal activities against Plutella xylostella and Aphis fabae in vivo. The structures of title compounds were well supported by spectroscopic data and single-crystal X-ray diffraction analysis. The compounds $\mathbf{6 d}$ and $\mathbf{6 w}$ showed outstanding activity against $P$. xylostella. And the compounds $6 \mathrm{e}, 6 \mathrm{l}, 6 \mathrm{~s}, 6 \mathrm{t}$ and $6 \mathrm{u}$ possessed good activity against $A$. fabae. The structure-activity relationship analysis demonstrated that the alkylene was linked at 3-position of benzothiazol-2-phenyl was beneficial to improve the bioactivity of title compounds against the aphids. Meanwhile, the compounds containing a alkylene linker with four carbon 
atoms would show better insecticidal activity. The results provided a practical guidance for further modification and optimization of the structures of title compounds.

\section{Experimental Section}

General. The melting points of the products were determined on a WRS-1B digital melting-point apparatus. NMR spectra were performed in DMSO- $d_{6}$ or $\mathrm{CDCl}_{3}$ on a Bruker Avance III 400 NMR spectrometer at room temperature with TMS as an internal standard. Thin-layer chromatography (TLC) was performed on silica gel GF254. High resolution mass spectrometry was recorded on an Agilent Technologies 6540 UHD Accurate-Mass $\mathrm{Q}-\mathrm{TOF}$ LC/MS. All reagents were analytical reagent grade pure and used directly without further purification.

Synthesis of coumarin intermediates 2. 7-Hydroxy coumarin $2 \mathrm{a}$ was synthesized according to the reported literature. ${ }^{20}$ Resorcinol $(11.0 \mathrm{~g}, 0.1 \mathrm{~mol})$ and 2-hydroxysuccinic acid $(20.1 \mathrm{~g}, 0.15 \mathrm{~mol})$ were added to a stirred solution of concentrated sulfuric acid $(200 \mathrm{~mL})$ in a reaction flask equipped with a thermometer and addition funnel. The reaction mixture was maintained at $100{ }^{\circ} \mathrm{C}$ for $2.5 \mathrm{~h}$ and poured into crushed ice under vigorous stirring. The off-white precipitate was filtered and purified by recrystallization from ethanol to give the product $\mathbf{2 a}$. Another coumarin intermediate 7-hydroxy-4-methyl-2H-chromen-2-one $\mathbf{2} \mathbf{b}$ was prepared under relatively mild conditions by condensing resorcinol with ethyl acetoacetate in the presence of a catalytic amount of concentrated sulfuric acid in 1,4-dioxane at $60^{\circ} \mathrm{C}$ for $4 \mathrm{~h}$. (see Supplementary file.)

Synthesis of benzothiazole intermediates 4. Sodium metabisulfite $(0.49 \mathrm{~g}, 2.5 \mathrm{mmol})$ was added to a DMF solution of 2-aminothiophenol $(2.50 \mathrm{~g}, 20 \mathrm{mmol})$ and 4-hydroxybenzaldehyde $(2.46 \mathrm{~g}, 20.2 \mathrm{mmol})$. The reaction mixture was stirred and heated to reflux for 2-2.5 $\mathrm{h}$. The progress of reaction was monitored periodically by TLC for completion. Then the mixture was poured into crushed ice, the formed precipitate was separated by filtration and washing to obtain 4-(benzothiazol-2-yl)phenol 4a. Other benzothiazole intermediates 4-(benzothiazol-2-yl)-2-methoxyphenol 4b and 3-(benzothiazol-2-yl)phenol 4c were prepared using a similar synthetic method. ${ }^{21}$ (see Supplementary file.)

Synthesis of bromoalkyl intermediates 5. 1,2-Dibromoethane (4.5 g, $24 \mathrm{mmol}$ ) was added to a solution of 4a $(1.36 \mathrm{~g}, 6 \mathrm{mmol})$ and $\mathrm{K}_{2} \mathrm{CO}_{3}(2.48 \mathrm{~g}, 18 \mathrm{mmmol})$ in acetone $(50 \mathrm{~mL})$. The mixture was stirred at reflux for 4-6 $\mathrm{h}$. The reaction solution was then filtered and concentrated under reduced pressure. The obtained viscous liquid was purified by column chromatography to give a yellow solid 2-[4-(2-bromoethoxy)phenyl]benzothiazole 5a. The above synthetic method was also used to prepare other bromoalkyl intermediates 5b-5o. (see Supplementary file.)

Synthesis of title compounds 6 . The mixture of $5 \mathbf{a}(0.54 \mathrm{~g}, 1.6 \mathrm{mmol}), 2 \mathrm{a}(0.26 \mathrm{~g}, 1.6 \mathrm{mmol})$ and anhydrous $\mathrm{K}_{2} \mathrm{CO}_{3}(0.41 \mathrm{~g}, 3 \mathrm{mmol})$ in DMF $(25 \mathrm{~mL})$ was heated at $60-70{ }^{\circ} \mathrm{C}$ for $6 \mathrm{~h}$. The reaction solution was then poured into crushed ice. The precipitate was filtered and purified by column chromatography with the mixed solution of ethyl acetate and petroleum ether ( $/ \mathrm{v} 1: 2)$ to yield the title compound 6a. The preparation method of target compounds $\mathbf{6 b}-\mathbf{6} \mathbf{d d}$ was similar to that of $6 \mathbf{a}$.

7-\{2-[4-(Benzothiazol-2-yl)phenoxy]ethoxy\}-2H-chromen-2-one (6a). White powder; yield 60.3\%; $\mathrm{mp} \mathrm{217-}$ $218{ }^{\circ} \mathrm{C} ;{ }^{1} \mathrm{H}$ NMR $\left(400 \mathrm{MHz}, \mathrm{DMSO}-d_{6}\right) \delta: 8.11$ (t, J $8.1 \mathrm{~Hz}, 1 \mathrm{H}$, Benzothiazole-4-H), 8.09-7.95 (m, 4H, Benzothiazole-7-H, Benzothiazol-2-phenyl-2,6-2H, Coumarin-4-H), 7.66 (d, J $8.6 \mathrm{~Hz}, 1 \mathrm{H}$, Coumarin-5- $\mathrm{H}), 7.53$ (t, J $7.5 \mathrm{~Hz}, 1 \mathrm{H}$, Benzothiazole-5-H), 7.44 (t, J $7.4 \mathrm{~Hz}, 1 \mathrm{H}$, Benzothiazole-6-H), 7.19 (d, J 8.6 Hz, 2H, Benzothiazol-2phenyl-3,5-2H), 7.10 (s, 1H, Coumarin-8-H), $7.03(\mathrm{~d}, J 8.5 \mathrm{~Hz}, 1 \mathrm{H}$, Coumarin-6-H), $6.32(\mathrm{~d}, J 9.5 \mathrm{~Hz}, 1 \mathrm{H}$, Coumarin-3-H), 4.49 (s, 4H,2 x CH 2$\left.) ;{ }^{13} \mathrm{C} \mathrm{NMR} \mathrm{(101} \mathrm{MHz,} \mathrm{CDCl}_{3}\right) \delta: 167.6,161.7,161.0,160.7,155.8,154.1$, 
143.3, 134.8, 129.1, 128.8, 127.0, 126.2, 124.9, 122.8, 121.5, 116.9, 115.0, 113.4, 113.0, 112.9, 101.6, 66.9, 66.3; HRMS (ESI) $m / z$ for $\mathrm{C}_{24} \mathrm{H}_{18} \mathrm{NO}_{4} \mathrm{~S}[\mathrm{M}+\mathrm{H}]^{+}$calcd. 416.0957, found. 416.0948 .

7-\{3-[4-(Benzothiazol-2-yl)phenoxy]propoxy\}-2H-chromen-2-one (6b). White powder; yield 64.5\%; $\mathrm{mp} \mathrm{175-}$ $176{ }^{\circ} \mathrm{C} ;{ }^{1} \mathrm{H}$ NMR (400 MHz, DMSO-d 6$) \delta: 8.12$ (d, J $7.9 \mathrm{~Hz}, 1 \mathrm{H}$, Benzothiazole-4-H), 8.07-7.96 (m, 4H, Benzothiazole-7-H, Benzothiazol-2-phenyl-2,6-2H, Coumarin-4- $\mathrm{H}), 7.64(\mathrm{~d}, \mathrm{~J} 8.6 \mathrm{~Hz}, 1 \mathrm{H}$, Coumarin-5- $\mathrm{H}), 7.53(\mathrm{t}$, J $7.2 \mathrm{~Hz}, 1 \mathrm{H}$, Benzothiazole-5-H), 7.43 (t, J $7.6 \mathrm{~Hz}, 1 \mathrm{H}$, Benzothiazole-6-H), 7.16 (d, J $8.8 \mathrm{~Hz}, 2 \mathrm{H}$, Benzothiazol-2phenyl-3,5-2H), 7.05 (d, J $2.2 \mathrm{~Hz}, 1 \mathrm{H}$, Coumarin-8-H), $7.00(\mathrm{dd}, J 8.6,2.3 \mathrm{~Hz}, 1 \mathrm{H}$, Coumarin-6-H), $6.30(\mathrm{~d}, J 9.5$ $\mathrm{Hz}, 1 \mathrm{H}$, Coumarin-3-H), 4.27 (dd, J 14.1, $\left.6.3 \mathrm{~Hz}, 4 \mathrm{H}, 2 \times \mathrm{CH}_{2}\right), 2.29-2.20\left(\mathrm{~m}, 2 \mathrm{H}, \mathrm{CH}_{2}\right) ;{ }^{13} \mathrm{C} \mathrm{NMR}\left(101 \mathrm{MHz}, \mathrm{CDCl}_{3}\right)$ $\delta: 167.7,162.0,161.1,161.0,155.8,154.2,143.3,134.8,129.1,128.8,126.6,126.2,124.8,122.8,121.5,114.8$, 113.1, 112.8, 112.6, 101.5, 64.9, 64.3, 28.9; HRMS (ESI) $\mathrm{m} / z$ for $\mathrm{C}_{25} \mathrm{H}_{20} \mathrm{NO}_{4} \mathrm{~S}[\mathrm{M}+\mathrm{H}]^{+}$calcd. 430.1113, found. 430.1104.

7-\{4-[4-(Benzothiazol-2-yl)phenoxy]butoxy\}-2H-chromen-2-one (6c).White powder; yield 60.5\%; mp 158-160 ${ }^{\circ} \mathrm{C} ;{ }^{1} \mathrm{H}$ NMR $\left(400 \mathrm{MHz}, \mathrm{DMSO}-d_{6}\right) \delta: 8.11(\mathrm{~d}, J 7.9 \mathrm{~Hz}, 1 \mathrm{H}$, Benzothiazole-4-H), 8.02 (dd, J 14.2, $9.0 \mathrm{~Hz}, 4 \mathrm{H}$, Benzothiazole-7-H, Benzothiazol-2-phenyl-2,6-2H, Coumarin-4-H), 7.63 (d, J 8.6 Hz, 1H, Coumarin-5-H), 7.53 (t, J 7.4 Hz, 1H, Benzothiazole-5-H), 7.43 (t, J 7.5 Hz, 1H, Benzothiazole-6-H), 7.13 (d, J 8.8 Hz, 2H, Benzothiazol-2phenyl-3,5-2H), 7.02 (d, J $2.0 \mathrm{~Hz}, 1 \mathrm{H}$, Coumarin-8-H), 6.97 (dd, J 8.6, 2.3 Hz, 1H, Coumarin-6-H), 6.29 (d, J 9.5 $\mathrm{Hz}, 1 \mathrm{H}$, Coumarin-3-H), 4.17 (d, J $\left.4.8 \mathrm{~Hz}, 4 \mathrm{H}, 2 \times \mathrm{CH}_{2}\right), 1.93\left(\mathrm{~s}, 4 \mathrm{H}, 2 \times \mathrm{CH}_{2}\right) ;{ }^{13} \mathrm{C} \mathrm{NMR}\left(101 \mathrm{MHz}, \mathrm{CDCl}_{3}\right) \delta: 167.8$, $162.2,161.2,155.9,154.2,143.4,134.8,129.1,128.7,126.4,126.2,124.8,122.8,121.5,114.8,113.0,112.9$, 112.5, 101.3, 68.1, 67.5, 25.8, 25.8; HRMS (ESI) $m / z$ for $\mathrm{C}_{26} \mathrm{H}_{22} \mathrm{NO}_{4} \mathrm{~S}[\mathrm{M}+\mathrm{H}]^{+}$calcd. 444.1270, found. 444.1260.

7-\{5-[4-(Benzothiazol-2-yl)phenoxy]pentyloxy\}-2H-chromen-2-one (6d). Grey powder; yield 68.4\%; mp 149$151{ }^{\circ} \mathrm{C}$; $\left.1 \mathrm{H} \mathrm{NMR} \mathrm{(400} \mathrm{MHz,} \mathrm{DMSO-d}\right) \delta: 8.11(\mathrm{t}, J 6.5 \mathrm{~Hz}, 1 \mathrm{H}$, Benzothiazole-4-H), 8.06-7.96 (m, 4H, Benzothiazole-7-H, Benzothiazol-2-phenyl-2,6-2H, Coumarin-4-H), 7.62 (d, J $8.6 \mathrm{~Hz}, 1 \mathrm{H}$, Coumarin-5-H), 7.52 (dd, J 11.2, $4.1 \mathrm{~Hz}, 1 \mathrm{H}$, Benzothiazole-5-H), 7.43 (t, J $7.1 \mathrm{~Hz}, 1 \mathrm{H}$, Benzothiazole-6-H), $7.12(\mathrm{~d}, J 8.8 \mathrm{~Hz}, 2 \mathrm{H}$, Benzothiazol-2-phenyl-3,5-2H), 7.00 (d, J $2.2 \mathrm{~Hz}, 1 \mathrm{H}$, Coumarin-8-H), 6.96 (dd, J 8.6, 2.3 Hz, 1H, Coumarin-6-H), $6.28\left(\mathrm{~d}, J 9.5 \mathrm{~Hz}, 1 \mathrm{H}\right.$, Coumarin-3-H), 4.11 (dd, J 10.4, $6.2 \mathrm{~Hz}, 4 \mathrm{H}, 2 \times \mathrm{CH}_{2}$ ), $1.83\left(\mathrm{dt}, J 13.4,6.5 \mathrm{~Hz}, 4 \mathrm{H}, 2 \times \mathrm{CH}_{2}\right)$, 1.60 (dt, J 14.8, 7.4 Hz, 2H, CH ); 13C NMR (101 MHz, CDCl $)_{3} \delta: 167.8,162.2,161.3,161.2,155.8,154.2,143.4$, $134.8,129.0,128.7,126.2,126.2,124.8,122.7,121.5,114.8,112.8,112.4,101.3,68.3,67.8,28.8,28.7,22.6$; HRMS (ESI) $m / z$ for $\mathrm{C}_{27} \mathrm{H}_{24} \mathrm{NO}_{4} \mathrm{~S}[\mathrm{M}+\mathrm{H}]^{+}$calcd. 458.1426, found. 458.1420 .

7-\{6-[4-(Benzothiazol-2-yl)phenoxy]hexyloxy\}-2H-chromen-2-one (6e). Grey powder; yield 57.8\%; mp 143$144{ }^{\circ} \mathrm{C} ;{ }^{1} \mathrm{H}$ NMR (400 MHz, DMSO-d 6$) \delta: 8.11$ (d, J $7.9 \mathrm{~Hz}, 1 \mathrm{H}$, Benzothiazole-4-H), 8.06-7.94 (m, 4H, Benzothiazole-7-H, Benzothiazol-2-phenyl-2,6-2H, Coumarin-4-H), 7.62 (d, J $8.6 \mathrm{~Hz}, 1 \mathrm{H}$, Coumarin-5-H), 7.52 (t, J $7.6 \mathrm{~Hz}, 1 \mathrm{H}$, Benzothiazole-5-H), 7.43 (t, J $7.5 \mathrm{~Hz}, 1 \mathrm{H}$, Benzothiazole-6-H), 7.11 (d, J 8.7 Hz, 2H, Benzothiazol-2phenyl-3,5-2H), $6.98(\mathrm{~s}, 1 \mathrm{H}$, Coumarin-8-H), $6.97-6.92(\mathrm{~m}, 1 \mathrm{H}$, Coumarin-6-H), $6.28(\mathrm{~d}, J 9.5 \mathrm{~Hz}, 1 \mathrm{H}$, Coumarin3-H), 4.08 (d, J $\left.3.7 \mathrm{~Hz}, 4 \mathrm{H}, 2 \times \mathrm{CH}_{2}\right), 1.78\left(\mathrm{~s}, 4 \mathrm{H}, 2 \times \mathrm{CH}_{2}\right), 1.50\left(\mathrm{~s}, 4 \mathrm{H}, 2 \times \mathrm{CH}_{2}\right) ;{ }^{13} \mathrm{C} \mathrm{NMR}\left(101 \mathrm{MHz}^{\mathrm{C} C D C l}\right) \delta$ : 167.9, 162.3, 161.4, 161.2, 155.9, 154.2, 143.4, 134.8, 129.1, 128.7, 126.2, 126.2, 124.7, 122.7, 121.5, 114.8, $112.9(\mathrm{~d}, J=1.9 \mathrm{~Hz}), 112.4,101.3,68.4,67.9,29.0,28.9,25.8,25.7 ; \mathrm{HRMS}(\mathrm{ESI}) \mathrm{m} / \mathrm{z}$ for $\mathrm{C}_{28} \mathrm{H}_{26} \mathrm{NO}_{4} \mathrm{~S}[\mathrm{M}+\mathrm{H}]^{+}$ calcd. 472.1583, found. 472.1576.

7-\{2-[4-(Benzothiazol-2-yl)phenoxy]ethoxy\}-4-methyl-2H-chromen-2-one (6f). White powder; yield 62.3\%; mp 227-228 ${ }^{\circ} \mathrm{C} ;{ }^{1} \mathrm{H}$ NMR (400 MHz, DMSO-d $)_{\text {) }} \delta: 8.13$ (d, J 7.8 Hz, 1H, Benzothiazole-4-H), 8.04 (dd, J 15.4, 8.3 $\mathrm{Hz}, 3 \mathrm{H}$, Benzothiazole-7-H, Benzothiazol-2-phenyl-2,6-2H), 7.72 (d, J 8.7 Hz, 1H, Coumarin-5-H), 7.53 (t, J $7.5 \mathrm{~Hz}$, $1 \mathrm{H}$, Benzothiazole-5-H), $7.44(\mathrm{t}, J 7.5 \mathrm{~Hz}, 1 \mathrm{H}$, Benzothiazole-6- $\mathrm{H}), 7.21(\mathrm{t}, J 7.2 \mathrm{~Hz}, 2 \mathrm{H}$, Benzothiazol-2-phenyl3,5-2H), 7.10 (d, J $2.2 \mathrm{~Hz}, 1 \mathrm{H}$, Coumarin-8-H), 7.05 (d, J 8.7 Hz, 1H, Coumarin-6-H), 6.24 (s, 1H, Coumarin-3-H), $4.49\left(\mathrm{~s}, 4 \mathrm{H}, 2 \times \mathrm{CH}_{2}\right), 2.42\left(\mathrm{~s}, 3 \mathrm{H}, \mathrm{CH}_{3}\right) ;{ }^{13} \mathrm{C} \mathrm{NMR}\left(101 \mathrm{MHz}, \mathrm{CDCl}_{3}\right) \delta: 167.6,161.5,161.2,160.7,155.1,154.1$, $152.4,134.8,129.2,127.0,126.2,125.6,124.9,122.8,121.5,116.9,115.0,114.0,112.7,112.2,101.6,66.9$, 66.4, 18.6; HRMS (ESI) $m / z$ for $\mathrm{C}_{25} \mathrm{H}_{20} \mathrm{NO}_{4} \mathrm{~S}[\mathrm{M}+\mathrm{H}]^{+}$calcd. 430.1113 , found. 430.1105. 
7-\{3-[4-(Benzothiazol-2-yl)phenoxy]propoxy\}-4-methyl-2H-chromen-2-one (6g). White powder; yield 66.8\%; $\mathrm{mp} 129-132{ }^{\circ} \mathrm{C} ;{ }^{1} \mathrm{H}$ NMR (400 MHz, DMSO- $\left.d_{6}\right) \delta: 8.11$ (d, J $7.8 \mathrm{~Hz}, 1 \mathrm{H}$, Benzothiazole-4-H), $8.02(\mathrm{t}, J 8.4 \mathrm{~Hz}, 3 \mathrm{H}$, Benzothiazole-7-H, Benzothiazol-2-phenyl-2,6-2H), 7.68 (d, J $8.7 \mathrm{~Hz}, 1 \mathrm{H}$, Coumarin-5-H), $7.52(\mathrm{t}, J 7.2 \mathrm{~Hz}, 1 \mathrm{H}$, Benzothiazole-5-H), 7.43 (t, J $7.3 \mathrm{~Hz}, 1 \mathrm{H}$, Benzothiazole-6-H), 7.15 (d, J $8.8 \mathrm{~Hz}, 2 \mathrm{H}$, Benzothiazol-2-phenyl-3,52H), 7.05-6.95 (m, 2H, Coumarin-6,8-2H), $6.21\left(\mathrm{~s}, 1 \mathrm{H}\right.$, Coumarin-3-H), $4.27\left(\mathrm{dd}, J 13.3,6.4 \mathrm{~Hz}, 4 \mathrm{H}, 2 \times \mathrm{CH}_{2}\right)$, $2.39\left(\mathrm{~s}, 3 \mathrm{H}, \mathrm{CH}_{3}\right), 2.29-2.17\left(\mathrm{~m}, 2 \mathrm{H}, \mathrm{CH}_{2}\right) ;{ }^{13} \mathrm{C} \mathrm{NMR}\left(101 \mathrm{MHz}, \mathrm{CDCl}_{3}\right) \delta: 167.7,161.8,161.2,161.1,155.2,154.1$, $152.5,134.8,129.1,126.5,126.2,125.5,124.8,122.8,121.5,114.8,113.6,112.4,111.9,101.5,64.8,64.3,28.9$, 18.6; HRMS (ESI) $m / z$ for $\mathrm{C}_{26} \mathrm{H}_{22} \mathrm{NO}_{4} \mathrm{~S}[\mathrm{M}+\mathrm{H}]^{+}$calcd. 444.1270, found. 444.1260.

7-\{4-[4-(Benzothiazol-2-yl)phenoxy]butoxy\}-4-methyl-2H-chromen-2-one (6h). White powder; yield 67.5\%; mp 175-178 ${ }^{\circ} \mathrm{C} ;{ }^{1} \mathrm{H}$ NMR (400 MHz, DMSO-d $) \delta: 8.12(\mathrm{~d}, J 7.9 \mathrm{~Hz}, 1 \mathrm{H}$, Benzothiazole-4-H), $8.02(\mathrm{t}, J 7.9 \mathrm{~Hz}, 3 \mathrm{H}$, Benzothiazole-7-H, Benzothiazol-2-phenyl-2,6-2H), 7.68 (d, J 8.7 Hz, 1H, Coumarin-5-H), 7.52 (dd, J 11.2, 4.1 Hz, $1 \mathrm{H}$, Benzothiazole-5-H), 7.43 (t, J $7.6 \mathrm{~Hz}, 1 \mathrm{H}$, Benzothiazole-6- $\mathrm{H}$ ), 7.13 (d, J $8.8 \mathrm{~Hz}, 2 \mathrm{H}$, Benzothiazol-2-phenyl3,5-2H), 7.04-6.93 (m, 2H, Coumarin-6,8-2H), $6.21(\mathrm{~d}, J 0.9 \mathrm{~Hz}, 1 \mathrm{H}$, Coumarin-3-H), $4.16(\mathrm{t}, J 11.0 \mathrm{~Hz}, 4 \mathrm{H}, 2 \mathrm{x}$ $\left.\mathrm{CH}_{2}\right), 2.40\left(\mathrm{~s}, 3 \mathrm{H}, \mathrm{CH}_{3}\right), 1.93\left(\mathrm{~s}, 4 \mathrm{H}, 2 \times \mathrm{CH}_{2}\right) ;{ }^{13} \mathrm{C} \mathrm{NMR}\left(101 \mathrm{MHz}, \mathrm{CDCl}_{3}\right) \delta: 167.8,161.9,161.3,161.2,155.2$, $154.2,152.6,134.8,129.1,126.4,126.2,125.5,124.8,122.8,121.5,114.8,113.5,112.5,111.8,101.3,68.0$, 67.6, 25.8, 25.8, 18.6; HRMS (ESI) $\mathrm{m} / z$ for $\mathrm{C}_{27} \mathrm{H}_{24} \mathrm{NO}_{4} \mathrm{~S}[\mathrm{M}+\mathrm{H}]^{+}$calcd. 458.1426, found. 458.1419.

7-\{5-[4-(Benzothiazol-2-yl)phenoxy]pentyloxy\}-4-methyl-2H-chromen-2-one (6i). Grey powder; yield 54.5\%; mp 132-134 ${ }^{\circ} \mathrm{C} ;{ }^{1} \mathrm{H}$ NMR (400 MHz, DMSO-d $\left.)\right) \delta: 8.11$ (d, J $7.8 \mathrm{~Hz}, 1 \mathrm{H}$, Benzothiazole-4-H), 8.02 (dd, J 7.8, 4.3 $\mathrm{Hz}, 3 \mathrm{H}$, Benzothiazole-7-H, Benzothiazol-2-phenyl-2,6-2H), 7.67 (d, J 8.7 Hz, 1H, Coumarin-5-H), 7.52 (t, J $7.4 \mathrm{~Hz}$, $1 \mathrm{H}$, Benzothiazole-5-H), 7.43 (t, J $7.4 \mathrm{~Hz}, 1 \mathrm{H}$, Benzothiazole-6-H), 7.12 (d, J $8.7 \mathrm{~Hz}, 2 \mathrm{H}$, Benzothiazol-2-phenyl3,5-2H), 7.02-6.92 (m, 2H, Coumarin-6,8-2H), $6.20\left(\mathrm{~s}, 1 \mathrm{H}\right.$, Coumarin-3-H), 4.19-4.03 (m, 4H, $\left.2 \times \mathrm{CH}_{2}\right), 2.39(\mathrm{~s}$, $\left.3 \mathrm{H}, \mathrm{CH}_{3}\right), 1.92-1.76\left(\mathrm{~m}, 4 \mathrm{H}, 2 \times \mathrm{CH}_{2}\right), 1.68-1.54\left(\mathrm{~m}, 2 \mathrm{H}, \mathrm{CH}_{2}\right) ;{ }^{13} \mathrm{C} \mathrm{NMR}\left(101 \mathrm{MHz}, \mathrm{CDCl}_{3}\right) \delta: 167.8,162.0,161.3$, $161.3,155.2,154.1,152.6,134.8,129.0,126.2,126.2,125.5,124.8,122.7,121.5,114.8,113.4,112.5,111.7$, 101.3, 68.2, 67.8, 28.8, 28.7, 22.6, 18.6; HRMS (ESI) $\mathrm{m} / \mathrm{z}$ for $\mathrm{C}_{28} \mathrm{H}_{26} \mathrm{NO}_{4} \mathrm{~S}[\mathrm{M}+\mathrm{H}]^{+}$calcd. 472.1583, found. 472.1576.

7-\{6-[4-(Benzothiazol-2-yl)phenoxy]hexyloxy\}-4-methyl-2H-chromen-2-one (6j). Yellow-brown powder; yield 50.2\%; mp 156-158 ${ }^{\circ} \mathrm{C} ;{ }^{1} \mathrm{H}$ NMR (400 MHz, DMSO-d6) $\delta: 8.11$ (d, J 7.6 Hz, 1H, Benzothiazole-4-H), 7.99 (d, J $21.0 \mathrm{~Hz}, 3 \mathrm{H}$, Benzothiazole-7-H, Benzothiazol-2-phenyl-2,6-2H), 7.67 (d, J $8.3 \mathrm{~Hz}, 1 \mathrm{H}$, Coumarin-5-H), 7.52 (d, J $7.5 \mathrm{~Hz}, 1 \mathrm{H}$, Benzothiazole-5-H), 7.44 (d, J $7.4 \mathrm{~Hz}, 1 \mathrm{H}$, Benzothiazole-6-H), 7.11 (d, J $8.0 \mathrm{~Hz}, 2 \mathrm{H}$, Benzothiazol-2phenyl-3,5-2H), $6.96\left(\mathrm{~d}, J 11.0 \mathrm{~Hz}, 2 \mathrm{H}\right.$, Coumarin-6,8-2H), $6.20\left(\mathrm{~s}, 1 \mathrm{H}\right.$, Coumarin-3-H), $4.09\left(\mathrm{~s}, 4 \mathrm{H}, 2 \times \mathrm{CH}_{2}\right), 2.39$ $\left(\mathrm{s}, 3 \mathrm{H}, \mathrm{CH}_{3}\right), 1.72\left(\mathrm{~s}, 4 \mathrm{H}, 2 \times \mathrm{CH}_{2}\right), 1.47\left(\mathrm{~s}, 4 \mathrm{H}, 2 \times \mathrm{CH}_{2}\right) ;{ }^{13} \mathrm{C} \mathrm{NMR}\left(101 \mathrm{MHz}, \mathrm{CDCl}_{3}\right) \delta: 167.8,162.1,161.4,161.3$, $155.2,154.2,152.6,134.8,129.0,126.2$, 126.2, 125.5, 124.7, 122.7, 121.5, 114.8, 113.4, 112.6, 111.7, 101.3, 68.3, 67.9, 29.0, 28.9, 25.7, 25.7, 18.6; HRMS (ESI) $\mathrm{m} / \mathrm{z}$ for $\mathrm{C}_{29} \mathrm{H}_{28} \mathrm{NO}_{4} \mathrm{~S}[\mathrm{M}+\mathrm{H}]^{+}$calcd. 486.1739, found. 486.1735.

7-\{2-[4-(Benzothiazol-2-yl)-2-methoxyphenoxy]ethoxy\}-2H-chromen-2-one (6k). White powder; yield 63.7\%; mp 207-208 ${ }^{\circ} \mathrm{C} ;{ }^{1} \mathrm{H}$ NMR (400 MHz, DMSO- $\left.d_{6}\right) \delta: 8.12(\mathrm{~d}, J 8.1 \mathrm{~Hz}, 1 \mathrm{H}$, Benzothiazole-4-H), $8.03(\mathrm{t}, J 8.1 \mathrm{~Hz}, 2 \mathrm{H}$, Benzothiazole-7- $\mathrm{H}$, Coumarin-4-H), 7.71-7.60 (m, 3H, Coumarin-5- $\mathrm{H}$, Benzothiazole-5,6-2H), $7.54(\mathrm{t}, J 7.1 \mathrm{~Hz}$, $1 \mathrm{H}$, Benzothiazol-2-phenyl-6-H), $7.44(\mathrm{t}, J 7.6 \mathrm{~Hz}, 1 \mathrm{H}$, Benzothiazol-2-phenyl-2-H), $7.22(\mathrm{~d}, J 8.4 \mathrm{~Hz}, 1 \mathrm{H}$, Coumarin-8-H), 7.13 (d, J $2.3 \mathrm{~Hz}, 1 \mathrm{H}$, Coumarin-6-H), 7.03 (dd, J 8.6, 2.4 Hz, 1H, Benzothiazol-2-phenyl-5-H), $6.32\left(\mathrm{~d}\right.$, J $9.5 \mathrm{~Hz}, 1 \mathrm{H}$, Coumarin-3-H), 4.50 (d, J $\left.5.1 \mathrm{~Hz}, 2 \mathrm{H}, \mathrm{CH}_{2}\right), 4.45$ (d, J $\left.4.9 \mathrm{~Hz}, 2 \mathrm{H}, \mathrm{CH}_{2}\right), 3.90\left(\mathrm{~s}, 3 \mathrm{H}, \mathrm{OCH}_{3}\right) ;{ }^{13} \mathrm{C}$ NMR $\left(101 \mathrm{MHz}, \mathrm{CDCl}_{3}\right) \delta: 167.7,161.8,161.1,155.8,154.1,150.4,149.9,143.3,134.9,128.8,127.6,126.3$, 125.0, 122.9, 121.5, 120.98, 113.5, 113.4, 113.1, 112.9, 110.4, 101.8, 67.5, 67.0, 56.1; HRMS (ESI) $\mathrm{m} / \mathrm{z}$ for $\mathrm{C}_{25} \mathrm{H}_{20} \mathrm{NO}_{5} \mathrm{~S}[\mathrm{M}+\mathrm{H}]^{+}$calcd. 446.1062, found. 446.1054.

7-\{3-[4-(Benzothiazol-2-yl)-2-methoxyphenoxy]propoxy\}-2H-chromen-2-one (6I). White powder; yield 61.9\%; $\mathrm{mp} 161-163{ }^{\circ} \mathrm{C} ;{ }^{1} \mathrm{H}$ NMR $\left(400 \mathrm{MHz}, \mathrm{DMSO}-d_{6}\right) \delta: 8.11$ (d, J 7.8 Hz, 1H, Benzothiazole-4-H), 8.01 (dd, J 14.6, 8.8 
$\mathrm{Hz}, 2 \mathrm{H}$, Benzothiazole-7-H, Coumarin-4-H), 7.62 (dd, J 14.3, $11.0 \mathrm{~Hz}, 3 \mathrm{H}$, Coumarin-5-H, Benzothiazole-5,6-2H), 7.53 (t, J $7.5 \mathrm{~Hz}, 1 \mathrm{H}$, Benzothiazol-2-phenyl-6-H), 7.43 (t, J 7.4 Hz, 1H, Benzothiazol-2-phenyl-2-H), 7.16 (d, J 8.4 $\mathrm{Hz}, 1 \mathrm{H}$, Coumarin-8-H), 7.05 (s, 1H, Coumarin-6-H), 6.99 (d, J $8.6 \mathrm{~Hz}, 1 \mathrm{H}$, Benzothiazol-2-phenyl-5-H), 6.29 (d, J $9.5 \mathrm{~Hz}, 1 \mathrm{H}$, Coumarin-3-H), 4.32-4.18 (m, 4H, $\left.2 \times \mathrm{CH}_{2}\right), 3.91\left(\mathrm{~s}, 3 \mathrm{H}, \mathrm{OCH}_{3}\right), 2.30-2.18\left(\mathrm{~m}, 2 \mathrm{H}, \mathrm{CH}_{2}\right) ;{ }^{13} \mathrm{C} \mathrm{NMR}(101$ $\left.\mathrm{MHz}_{1} \mathrm{CDCl}_{3}\right) \delta: 167.8,162.0,161.2,155.8,154.1,150.7,149.7,143.3,134.9,128.7,127.0,126.2,124.9,122.8$, $121.5,121.0,113.1,112.8,112.7,112.6,110.2,101.5,65.3,64.9,56.1,28.9$. HRMS (ESI) $m / z$ for $\mathrm{C}_{26} \mathrm{H}_{22} \mathrm{NO}_{5} \mathrm{~S}$ $[\mathrm{M}+\mathrm{H}]^{+}$calcd. 460.1219 , found, 460.1209 .

7-\{4-[4-(Benzothiazol-2-yl)-2-methoxyphenoxy]butoxy\}-2H-chromen-2-one (6m). White powder; yield 58.1\%; $\mathrm{mp} 142-143{ }^{\circ} \mathrm{C} ;{ }^{1} \mathrm{H}$ NMR (400 MHz, DMSO-d $\left.)\right) \delta: 8.11$ (d, J 7.9 Hz, 1H, Benzothiazole-4-H), 8.01 (dd, J 14.9, 8.8 $\mathrm{Hz}, 2 \mathrm{H}$, Benzothiazole-7-H, Coumarin-4-H), 7.68-7.57 (m, 3H, Coumarin-5- H, Benzothiazole-5,6-2H), $7.53(\mathrm{t}, \mathrm{J}$ $7.7 \mathrm{~Hz}, 1 \mathrm{H}$, Benzothiazol-2-phenyl-6-H), 7.43 (t, J $7.6 \mathrm{~Hz}, 1 \mathrm{H}$, Benzothiazol-2-phenyl-2-H), 7.15 (d, J 8.4 Hz, 1H, Coumarin-8-H), 7.01(s, 1H, Coumarin-6-H), $6.97(\mathrm{~d}, J 8.6 \mathrm{~Hz}, 1 \mathrm{H}$, Benzothiazol-2-phenyl-5-H), 6.29 (d, J $9.5 \mathrm{~Hz}$, $1 \mathrm{H}$, Coumarin-3-H), 4.17 (d, J $\left.15.5 \mathrm{~Hz}, 4 \mathrm{H}, 2 \times \mathrm{CH}_{2}\right), 3.90\left(\mathrm{~s}, 3 \mathrm{H}, \mathrm{OCH}_{3}\right), 1.94\left(\mathrm{~s}, 4 \mathrm{H}, 2 \times \mathrm{CH}_{2}\right) ;{ }^{13} \mathrm{C} \mathrm{NMR}(101 \mathrm{MHz}$, $\mathrm{CDCl}_{3}$ ) $\delta: 167.8,162.2,161.2,155.8,154.1,150.9,149.6,143.4,134.9,128.7,126.7,126.2,124.8,122.8,121.5$, 121.0, 112.9, 112.9, 112.4, 112.3, 110.1, 101.3, 68.5, 68.1, 56.1, 25.9, 25.7; HRMS (ESI) $\mathrm{m} / z$ for $\mathrm{C}_{27} \mathrm{H}_{24} \mathrm{NO}_{5} \mathrm{~S}$ $[\mathrm{M}+\mathrm{H}]^{+}$calcd. 474.1375 , found, 474.1370 .

7-\{5-[4-(Benzothiazol-2-yl)-2-methoxyphenoxy]pentyloxy\}-2H-chromen-2-one (6n). White powder; yield 66.3\%; mp 116-118 ${ }^{\circ} \mathrm{C} ;{ }^{1} \mathrm{H}$ NMR (400 MHz, DMSO- $\left.d_{6}\right) \delta: 8.10$ (t, J $6.5 \mathrm{~Hz}, 1 \mathrm{H}$, Benzothiazole-4-H), 8.06-7.98 (m, $1 \mathrm{H}$, Benzothiazole-7-H), 7.97 (d, J $6.5 \mathrm{~Hz}, 1 \mathrm{H}$, Coumarin-4-H), 7.61 (ddd, J 10.3, 7.4, 2.0 Hz, 3H, Coumarin-5-H, Benzothiazole-5,6-2H), $7.53(\mathrm{t}, J 7.7 \mathrm{~Hz}, 1 \mathrm{H}$, Benzothiazol-2-phenyl-6-H), 7.43 (t, J $7.6 \mathrm{~Hz}, 1 \mathrm{H}$, Benzothiazol-2phenyl-2-H), 7.14 (d, J 8.4 Hz, 1H, Coumarin-8-H), 6.99 (dd, J 8.7, 3.6 Hz, 1H, Coumarin-6-H), 6.96 (dd, J 8.6, 2.4 $\mathrm{Hz}, 1 \mathrm{H}$, Benzothiazol-2-phenyl-5-H), 6.28 (d, J $9.5 \mathrm{~Hz}, 1 \mathrm{H}$, Coumarin-3-H), 4.18-3.99 (m, 4H, $\left.2 \times \mathrm{CH}_{2}\right), 3.90(\mathrm{~s}$, $\left.3 \mathrm{H}, \mathrm{OCH}_{3}\right), 1.92-1.74\left(\mathrm{~m}, 4 \mathrm{H}, 2 \times \mathrm{CH}_{2}\right), 1.60\left(\mathrm{dt}, J 15.1,7.7 \mathrm{~Hz}, 2 \mathrm{H}, \mathrm{CH}_{2}\right) ;{ }^{13} \mathrm{C} \mathrm{NMR}\left(101 \mathrm{MHz}, \mathrm{CDCl}_{3}\right) \delta: 167.9$, 162.3, 161.2, 155.9, 154.1, 151.1, 149.6, 143.4, 134.9, 128.7, 126.5, 126.2, 124.8, 122.8, 121.5, 121.1, 112.9, 112.4, 112.3, 110.1, 101.3, 68.8, 68.4, 56.2, 29.0, 28.9, 25.7; HRMS (ESI) $\mathrm{m} / \mathrm{z}$ for $\mathrm{C}_{28} \mathrm{H}_{26} \mathrm{NO}_{5} \mathrm{~S}[\mathrm{M}+\mathrm{H}]^{+} \mathrm{calcd}$. 488.1532, found, 488.1523.

7-\{6-[4-(Benzothiazol-2-yl)-2-methoxyphenoxy]hexyloxy\}-2H-chromen-2-one (6o). Yellow-white powder; yield 46.5\%; mp 125-126 ${ }^{\circ} \mathrm{C} ;{ }^{1} \mathrm{H}$ NMR (400 MHz, DMSO- $\left.d_{6}\right) \delta: 8.11$ (d, J 7.9 Hz, $1 \mathrm{H}$, Benzothiazole-4-H), 8.01 (dd, J 16.6, 8.8 Hz, 2H, Benzothiazole-7-H, Coumarin-4-H), 7.70-7.56 (m, 3H, Coumarin-5-H, Benzothiazole-5,6-2H), $7.53(\mathrm{t}, J 7.3 \mathrm{~Hz}, 1 \mathrm{H}$, Benzothiazol-2-phenyl-6-H), 7.43 (t, J 7.5 Hz, 1H, Benzothiazol-2-phenyl-2-H), 7.13 (d, J 8.4 $\mathrm{Hz}, 1 \mathrm{H}$, Coumarin-8-H), 6.99 (d, J $2.1 \mathrm{~Hz}, 1 \mathrm{H}$, Coumarin-6-H), 6.95 (dd, J 8.6, $2.3 \mathrm{~Hz}, 1 \mathrm{H}$, Benzothiazol-2-phenyl5-H), 6.28 (d, J $9.5 \mathrm{~Hz}, 1 \mathrm{H}$, Coumarin-3-H), 4.07 (dq, J 12.2, $\left.6.8 \mathrm{~Hz}, 4 \mathrm{H}, 2 \times \mathrm{CH}_{2}\right), 3.89\left(\mathrm{~s}, 3 \mathrm{H}, \mathrm{OCH}_{3}\right), 1.79(\mathrm{~d}, J 5.2$ $\left.\mathrm{Hz}, 4 \mathrm{H}, 2 \times \mathrm{CH}_{2}\right), 1.51\left(\mathrm{~s}, 4 \mathrm{H}, 2 \times \mathrm{CH}_{2}\right) ;{ }^{13} \mathrm{C} \mathrm{NMR}\left(101 \mathrm{MHz}, \mathrm{CDCl}_{3}\right) \delta: 167.9,162.5,162.2,161.2,155.8,154.1$, 151.0, 149.6, 143.4, 134.8, 128.7, 126.5, 126.2, 124.8, 122.7, 121.5, 121.1, 112.8, 112.4, 110.1, 101.3, 68.7, 68.3, 56.1, 36.4, 31.4, 28.7, 22.6; HRMS (ESI) $\mathrm{m} / \mathrm{z}$ for $\mathrm{C}_{29} \mathrm{H}_{28} \mathrm{NO}_{5} \mathrm{~S}[\mathrm{M}+\mathrm{H}]^{+}$calcd. 502.1688, found, 502.1680.

7-\{2-[4-(Benzothiazol-2-yl)-2-methoxyphenoxy]ethoxy\}-4-methyl-2H-chromen-2-one (6p). White powder; yield 68.4\%; mp 218-219 ${ }^{\circ} \mathrm{C} ;{ }^{1} \mathrm{H}$ NMR (400 MHz, DMSO- $d_{6}$ ) $\delta: 8.12$ (d, J 7.9 Hz, $1 \mathrm{H}$, Benzothiazole-4-H), 8.04 (d, J $8.1 \mathrm{~Hz}, 1 \mathrm{H}$, Benzothiazole-7-H), 7.74-7.60 (m, 3H, Coumarin-5-H, Benzothiazole-5,6-2H), 7.54 (t, J 7.6 Hz, 1H, Benzothiazol-2-phenyl-6-H), 7.44 (t, J 7.6 Hz, 1H, Benzothiazol-2-phenyl-2-H), 7.23 (t, J 7.8 Hz, 1H, Coumarin-8H), $7.12(\mathrm{~d}, J 2.4 \mathrm{~Hz}, 1 \mathrm{H}$, Coumarin-6-H), 7.05 (dd, J 8.8, 2.4 Hz, 1H, Benzothiazol-2-phenyl-5-H), 6.24 (s, 1H, Coumarin-3-H), 4.53-4.42 (m, 4H, $\left.2 \times \mathrm{CH}_{2}\right), 3.90\left(\mathrm{~s}, 3 \mathrm{H}, \mathrm{OCH}_{3}\right), 2.42\left(\mathrm{~s}, 3 \mathrm{H}, \mathrm{CH}_{3}\right) ;{ }^{13} \mathrm{C} \mathrm{NMR}\left(101 \mathrm{MHz}, \mathrm{CDCl}_{3}\right) \delta$ : 167.7, 161.6, 161.2, 155.2, 154.1, 152.5, 150.4, 149.9, 134.9, 127.6, 126.3, 125.5, 125.0, 122.9, 121.5, 120.9, $113.9,113.5,112.7,112.2,110.4,101.8,67.5,67.0,56.1,18.6$; HRMS (ESI) $\mathrm{m} / \mathrm{z}$ for $\mathrm{C}_{26} \mathrm{H}_{22} \mathrm{NO}_{5} \mathrm{~S}[\mathrm{M}+\mathrm{H}]^{+} \mathrm{calcd}$. 460.1219 , found, 460.1208 . 
7-\{3-[4-(Benzothiazol-2-yl)-2-methoxyphenoxy]propoxy\}-4-methyl-2H-chromen-2-one (6q). White powder; yield 60.2\%; mp 156-158 ${ }^{\circ} \mathrm{C} ;{ }^{1} \mathrm{H}$ NMR (400 MHz, DMSO- $\left.d_{6}\right) \delta: 8.11$ (d, J 7.9 Hz, $1 \mathrm{H}$, Benzothiazole-4-H), 8.03 (d, J $8.1 \mathrm{~Hz}, 1 \mathrm{H}$, Benzothiazole-7-H), 7.71-7.63 (m, 2H, Coumarin-5-H, Benzothiazole-6-H), 7.60 (d, J 7.9 Hz, 1H, Benzothiazole-5-H), 7.53 (t, J $7.5 \mathrm{~Hz}, 1 \mathrm{H}$, Benzothiazol-2-phenyl-6-H), 7.43 (t, J $7.5 \mathrm{~Hz}, 1 \mathrm{H}$, Benzothiazol-2phenyl-2-H), 7.18 (d, J $8.4 \mathrm{~Hz}, 1 \mathrm{H}$, Coumarin-8-H), 7.04-6.97 (m, 2H, Coumarin-6- $\mathrm{H}$, Benzothiazol-2-phenyl-5- $\mathrm{H})$, $6.21\left(\mathrm{~s}, 1 \mathrm{H}\right.$, Coumarin-3-H), 4.32-4.20 (m, 4H, $\left.2 \times \mathrm{CH}_{2}\right), 3.91\left(\mathrm{~s}, 3 \mathrm{H}, \mathrm{OCH}_{3}\right), 2.39\left(\mathrm{~s}, 3 \mathrm{H}, \mathrm{CH}_{3}\right), 2.30-2.18(\mathrm{~m}, 2 \mathrm{H}$, $\left.\mathrm{CH}_{2}\right) ;{ }^{13} \mathrm{C}$ NMR $\left(101 \mathrm{MHz}, \mathrm{CDCl}_{3}\right) \delta: 167.8,161.8,161.2,155.2,154.1,152.5,150.8,149.7,134.8,126.9,126.2$, $125.5,124.9,122.8,121.5,121.0,113.6,112.7,112.5,111.9,110.2,101.5,65.3,64.9,56.1,28.9,18.6$; HRMS (ESI) $m / z$ for $\mathrm{C}_{27} \mathrm{H}_{24} \mathrm{NO}_{5} \mathrm{~S}[\mathrm{M}+\mathrm{H}]^{+}$calcd. 474.1375, found, 474.1374 .

7-\{4-[4-(Benzothiazol-2-yl)-2-methoxyphenoxy]butoxy\}-4-methyl-2H-chromen-2-one (6r). Off-white powder; yield 48.7\%; mp 123-124 ${ }^{\circ} \mathrm{C} ;{ }^{1} \mathrm{H}$ NMR (400 MHz, DMSO- $\left.d_{6}\right) \delta: 8.11$ (d, J 7.9 Hz, $1 \mathrm{H}$, Benzothiazole-4-H), 8.03 (d, J $8.2 \mathrm{~Hz}, 1 \mathrm{H}$, Benzothiazole-7-H), 7.71-7.63 (m, 2H, Coumarin-5- $\mathrm{H}$, Benzothiazole-6-H), $7.61(\mathrm{~d}, \mathrm{~J} 8.2 \mathrm{~Hz}, 1 \mathrm{H}$, Benzothiazole-5-H), 7.53 (t, J $7.6 \mathrm{~Hz}, 1 \mathrm{H}$, Benzothiazol-2-phenyl-6-H), 7.44 (t, J $7.5 \mathrm{~Hz}, 1 \mathrm{H}$, Benzothiazol-2phenyl-2-H), 7.15 (d, J $8.4 \mathrm{~Hz}, 1 \mathrm{H}$, Coumarin-8-H), 7.05-6.93 (m, 2H, Coumarin-6- $\mathrm{H}$, Benzothiazol-2-phenyl-5-H), $6.21\left(\mathrm{~s}, 1 \mathrm{H}\right.$, Coumarin-3-H), $4.15\left(\mathrm{t}, \mathrm{J} 16.7 \mathrm{~Hz}, 4 \mathrm{H}, 2 \times \mathrm{CH}_{2}\right), 3.90\left(\mathrm{~s}, 3 \mathrm{H}, \mathrm{OCH}_{3}\right), 2.40\left(\mathrm{~s}, 3 \mathrm{H}, \mathrm{CH}_{3}\right), 1.94(\mathrm{~s}, 4 \mathrm{H}, 2 \mathrm{x}$ $\left.\mathrm{CH}_{2}\right) ;{ }^{13} \mathrm{C} N M R\left(101 \mathrm{MHz} \mathrm{CDCl}_{3}\right) \delta: 167.9,162.0,161.3,155.2,154.1,152.6,150.9,149.6,134.9,126.7,126.2$, $125.5,124.9,122.8,121.5,121.0,113.5,112.6,112.4,111.8,110.1,101.3,68.5,68.1,56.1,25.9,25.7,18.6$; HRMS (ESI) $m / z$ for $\mathrm{C}_{28} \mathrm{H}_{26} \mathrm{NO}_{5} \mathrm{~S}[\mathrm{M}+\mathrm{H}]^{+}$calcd. 488.1532, found, 488.1523.

7-\{5-[4-(Benzothiazol-2-yl)-2-methoxyphenoxy]pentyloxy\}-4-methyl-2H-chromen-2-one (6s). White powder; yield 56.5\%; mp 126-128 ${ }^{\circ} \mathrm{C} ;{ }^{1} \mathrm{H}$ NMR (400 MHz, DMSO- $\left.d_{6}\right) \delta: 8.11$ (d, J 7.9 Hz, $1 \mathrm{H}$, Benzothiazole-4-H), 8.03 (d, J $8.1 \mathrm{~Hz}, 1 \mathrm{H}$, Benzothiazole-7-H), 7.73-7.62 (m, 2H, Coumarin-5- H, Benzothiazole-6-H), $7.58(\mathrm{t}, J 10.1 \mathrm{~Hz}, 1 \mathrm{H}$, Benzothiazole-5-H), $7.53(t, J 7.6 \mathrm{~Hz}, 1 \mathrm{H}$, Benzothiazol-2-phenyl-6- $\mathrm{H}), 7.43(\mathrm{t}, J 7.6 \mathrm{~Hz}, 1 \mathrm{H}$, Benzothiazol-2phenyl-2-H), 7.13 (d, J $8.3 \mathrm{~Hz}, 1 \mathrm{H}$, Coumarin-8-H), 6.97 (d, J $13.6 \mathrm{~Hz}, 2 \mathrm{H}$, Coumarin-6-H, Benzothiazol-2-phenyl$5-\mathrm{H}), 6.20\left(\mathrm{~s}, 1 \mathrm{H}\right.$, Coumarin-3-H), 4.15-4.06 (m, 4H, $\left.2 \times \mathrm{CH}_{2}\right), 3.90\left(\mathrm{~s}, 3 \mathrm{H}, \mathrm{OCH}_{3}\right), 2.38\left(\mathrm{~s}, 3 \mathrm{H}, \mathrm{CH}_{3}\right), 1.89-1.76(\mathrm{~m}$, $\left.4 \mathrm{H}, 2 \times \mathrm{CH}_{2}\right), 1.61$ (dd, J 14.5, 7.7 Hz, 2H, CH ); ${ }^{13} \mathrm{C} \mathrm{NMR} \mathrm{(101} \mathrm{MHz,} \mathrm{CDCl} 3$ ) $\delta: 167.9,162.0,161.3,155.2,154.1$, $152.5,151.0,149.6,134.8,126.5,126.2,125.4,124.8,122.8,121.5,121.1,113.4,112.5,112.4,111.8,110.1$, 101.3, 68.7, 68.2, 56.2, 28.7, 28.7, 22.6, 18.6; HRMS (ESI) $\mathrm{m} / \mathrm{z}$ for $\mathrm{C}_{29} \mathrm{H}_{28} \mathrm{NO}_{5} \mathrm{~S}[\mathrm{M}+\mathrm{H}]^{+}$calcd. 502.1688, found, 502.1681.

7-\{6-[4-(Benzothiazol-2-yl)-2-methoxyphenoxy]hexyloxy\}-4-methyl-2H-chromen-2-one (6t). White powder; yield 54.2\%; mp 118-119 ${ }^{\circ} \mathrm{C} ;{ }^{1} \mathrm{H}$ NMR (400 MHz, DMSO- $\left.d_{6}\right) \delta: 8.11$ (d, J 7.9 Hz, $1 \mathrm{H}$, Benzothiazole-4-H), 8.03 (d, J $8.1 \mathrm{~Hz}, 1 \mathrm{H}$, Benzothiazole-7-H), 7.70-7.62 (m, 2H, Coumarin-5- $\mathrm{H}$, Benzothiazole-6-H), 7.58 (t, J $9.9 \mathrm{~Hz}, 1 \mathrm{H}$, Benzothiazole-5-H), $7.53(\mathrm{t}, J 7.6 \mathrm{~Hz}, 1 \mathrm{H}$, Benzothiazol-2-phenyl-6-H), $7.43(\mathrm{t}, J 7.6 \mathrm{~Hz}, 1 \mathrm{H}$, Benzothiazol-2phenyl-2-H), 7.12 (d, J 8.4 Hz, 1H, Coumarin-8-H), 6.96 (d, J $9.8 \mathrm{~Hz}, 2 \mathrm{H}$, Coumarin-6- $\mathrm{H}$, Benzothiazol-2-phenyl5-H), 6.20 (s, 1H, Coumarin-3-H), 4.07 (dt, J 18.5, $9.2 \mathrm{~Hz}, 4 \mathrm{H}, 2 \times \mathrm{CH}_{2}$ ), $3.89\left(\mathrm{~s}, 3 \mathrm{H}, \mathrm{OCH}_{3}\right), 2.38\left(\mathrm{~s}, 3 \mathrm{H}, \mathrm{CH}_{3}\right), 1.79$ (d, J $\left.5.0 \mathrm{~Hz}, 4 \mathrm{H}, 2 \times \mathrm{CH}_{2}\right), 1.51$ (s, 4H, $\left.2 \times \mathrm{CH}_{2}\right) ;{ }^{13} \mathrm{C} \mathrm{NMR}\left(101 \mathrm{MHz}, \mathrm{CDCl}_{3}\right) \delta: 167.9,162.1,161.3,155.2,154.1$, $152.5,151.1,149.6,134.8,126.5,126.2$, 125.4, 124.8, 122.8, 121.5, 121.1, 113.4, 112.6, 112.3, 111.8, 110.1, 101.3, 68.8, 68.3, 56.2, 28.9, 28.9, 25.7, 25.7, 18.6; HRMS (ESI) $m / z$ for $\mathrm{C}_{30} \mathrm{H}_{30} \mathrm{NO}_{5} \mathrm{~S}[\mathrm{M}+\mathrm{H}]^{+}$calcd. 516.1845, found, 516.1842 .

7-\{2-[3-(Benzothiazol-2-yl)phenoxy]ethoxy\}-2H-chromen-2-one (6u). White powder; yield 71.2\%; mp 183$185{ }^{\circ} \mathrm{C} ;{ }^{1} \mathrm{H}$ NMR $\left(400 \mathrm{MHz}, \mathrm{DMSO}-d_{6}\right) \delta: 8.17(\mathrm{~d}, J 7.8 \mathrm{~Hz}, 1 \mathrm{H}$, Benzothiazole-4-H), $8.08(\mathrm{~d}, J 7.8 \mathrm{~Hz}, 1 \mathrm{H}$, Benzothiazole-7-H), 8.00 (t, J $14.0 \mathrm{~Hz}, 1 \mathrm{H}$, Coumarin-4-H), 7.73-7.63 (m, 3H, Benzothiazol-2-phenyl-6- $\mathrm{H}$, Coumarin-5-H, Benzothiazole-5-H), 7.61-7.41 (m, 3H, Benzothiazole-6- $\mathrm{H}$, Benzothiazol-2-phenyl-2,5-2H), 7.27$7.19(\mathrm{~m}, 1 \mathrm{H}$, Benzothiazol-2-phenyl-4-H), $7.11(\mathrm{~d}, J 2.3 \mathrm{~Hz}, 1 \mathrm{H}$, Coumarin-8-H), 7.03 (dt, J 12.7, $4.7 \mathrm{~Hz}, 1 \mathrm{H}$,

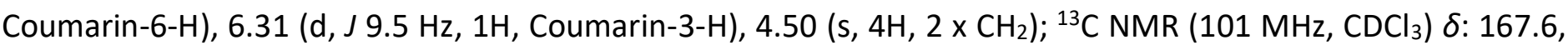
161.7, 161.1, 158.9, 155.8, 154.0, 143.3, 135.0, 135.0, 130.2, 128.8, 126.4, 125.3, 123.2, 121.6, 120.8, 118.0, 
113.4, 113.0, 112.9, 112.8, 101.6, 67.0, 66.4; HRMS (ESI) $\mathrm{m} / z$ for $\mathrm{C}_{24} \mathrm{H}_{18} \mathrm{NO}_{4} \mathrm{~S}[\mathrm{M}+\mathrm{H}]^{+}$calcd. 416.0957, found, 416.0949.

7-\{3-[3-(Benzothiazol-2-yl)phenoxy]propoxy\}-2H-chromen-2-one (6v). White powder; yield 56.2\%; mp 176$178{ }^{\circ} \mathrm{C} ;{ }^{1} \mathrm{H}$ NMR (400 MHz, DMSO-d $\left.)\right) \delta: 8.15(\mathrm{~d}, J 7.9 \mathrm{~Hz}, 1 \mathrm{H}$, Benzothiazole-4-H), $8.08(\mathrm{~d}, J 8.0 \mathrm{~Hz}, 1 \mathrm{H}$, Benzothiazole-7-H), 7.99 (d, J $9.5 \mathrm{~Hz}, 1 \mathrm{H}$, Coumarin-4-H), 7.64 (t, J $7.8 \mathrm{~Hz}, 3 \mathrm{H}$, Benzothiazol-2-phenyl-6-H, Coumarin-5-H, Benzothiazole-5-H), 7.56 (t, J 7.5 Hz, 1H, Benzothiazole-6-H), 7.53-7.43 (m, 2H, Benzothiazol-2phenyl-2,5-2H), 7.24-7.14 (m, 1H, Benzothiazol-2-phenyl-4-H), 7.09-6.93 (m, 2H, Coumarin-6,8-2H), 6.29 (d, J

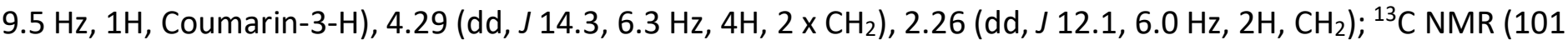
$\left.\mathrm{MHz}_{1} \mathrm{CDCl}_{3}\right) \delta: 167.8,162.0,161.2,159.2,155.8,154.0,143.4,135.0,134.9,130.1,128.7,126.3,125.2,123.2$, 121.6, 120.4, 117.7, 113.1, 112.8, 112.6, 101.5, 64.9, 64.3, 29.0; HRMS (ESI) $\mathrm{m} / z$ for $\mathrm{C}_{25} \mathrm{H}_{20} \mathrm{NO}_{4} \mathrm{~S}[\mathrm{M}+\mathrm{H}]^{+}$calcd. 430.1113 , found, 430.1112 .

7-\{4-[3-(Benzothiazol-2-yl)phenoxy]butoxy\}-2H-chromen-2-one (6w). White powder; yield 68.7\%; mp 123$125{ }^{\circ} \mathrm{C} ;{ }^{1} \mathrm{H}$ NMR $\left(400 \mathrm{MHz}, \mathrm{DMSO}-d_{6}\right) \delta: 8.15$ (t, J $7.0 \mathrm{~Hz}, 1 \mathrm{H}$, Benzothiazole-4-H), $8.07(\mathrm{~d}, J 8.1 \mathrm{~Hz}, 1 \mathrm{H}$, Benzothiazole-7-H), 7.99 (d, J 9.5 Hz, 1H, Coumarin-4-H), 7.68-7.59 (m, 3H, Benzothiazol-2-phenyl-6-H, Coumarin-5-H, Benzothiazole-5-H), 7.56 (t, J $7.6 \mathrm{~Hz}, 1 \mathrm{H}$, Benzothiazole-6-H), 7.49 (td, J 7.6, 3.9 Hz, 2H, Benzothiazol-2-phenyl-2,5-2H), 7.17 (d, J $6.0 \mathrm{~Hz}, 1 \mathrm{H}$, Benzothiazol-2-phenyl-4-H), 7.02 (s, 1H, Coumarin-8-H), $6.97(\mathrm{~d}$, J $8.6 \mathrm{~Hz}, 1 \mathrm{H}$, Coumarin-6-H), 6.28 (d, J $9.5 \mathrm{~Hz}, 1 \mathrm{H}$, Coumarin-3-H), 4.19 (s, 4H, 2 x CH 2$), 1.97$ (d, J 17.6 $\left.\mathrm{Hz}, 4 \mathrm{H}, 2 \times \mathrm{CH}_{2}\right) ;{ }^{13} \mathrm{CNMR}\left(101 \mathrm{MHz}, \mathrm{CDCl}_{3}\right) \delta: 167.9,162.2,161.2,159.3,155.9,154.0,143.4,135.0,134.9$, 130.0, 128.7, 126.3, 125.2, 123.2, 121.6, 120.2, 117.6, 113.0, 112.9, 112.7, 112.5, 101.4, 68.1, 67.6, 25.9, 25.8; HRMS (ESI) $m / z$ for $\mathrm{C}_{26} \mathrm{H}_{22} \mathrm{NO}_{4} \mathrm{~S}[\mathrm{M}+\mathrm{H}]^{+}$calcd. 444.1270, found, 444.1259.

7-\{5-[3-(Benzothiazol-2-yl)phenoxy]butoxy\}-2H-chromen-2-one (6x). White powder; yield 47.5\%; $\mathrm{mp} 143-$ $145{ }^{\circ} \mathrm{C} ;{ }^{1} \mathrm{H}$ NMR $\left(400 \mathrm{MHz}, \mathrm{DMSO}-d_{6}\right) \delta: 8.16(\mathrm{~d}, J 7.9 \mathrm{~Hz}, 1 \mathrm{H}$, Benzothiazole-4-H), $8.08(\mathrm{~d}, J 8.1 \mathrm{~Hz}, 1 \mathrm{H}$, Benzothiazole-7-H), 7.99 (d, J 9.5 Hz, 1H, Coumarin-4-H), 7.69-7.59 (m, 3H, Benzothiazol-2-phenyl-6-H, Coumarin-5-H, Benzothiazole-5-H), 7.56 (t, J $7.6 \mathrm{~Hz}, 1 \mathrm{H}$, Benzothiazole-6-H), 7.49 (dd, J 10.9, $5.0 \mathrm{~Hz}, 2 \mathrm{H}$, Benzothiazol-2-phenyl-2,5-2H), $7.16(\mathrm{~d}, J 8.2 \mathrm{~Hz}, 1 \mathrm{H}$, Benzothiazol-2-phenyl-4-H), 7.00 (s, 1H, Coumarin-8-H), $6.96\left(\mathrm{~d}\right.$, J $8.6 \mathrm{~Hz}, 1 \mathrm{H}$, Coumarin-6-H), $6.28\left(\mathrm{~d}, J 9.5 \mathrm{~Hz}, 1 \mathrm{H}\right.$, Coumarin-3-H), $4.13\left(\mathrm{t}, J 6.3 \mathrm{~Hz}, 4 \mathrm{H}, 2 \times \mathrm{CH}_{2}\right), 1.89-$ $1.78\left(\mathrm{~m}, 4 \mathrm{H}, 2 \times \mathrm{CH}_{2}\right), 1.67-1.57\left(\mathrm{~m}, 2 \mathrm{H}, \mathrm{CH}_{2}\right) ;{ }^{13} \mathrm{C} \mathrm{NMR}\left(101 \mathrm{MHz}, \mathrm{CDCl}_{3}\right) \delta: 168.0,162.3,161.2,159.5,155.9$, $153.9,143.4,135.0,134.8,130.0,128.7,126.3,125.2,123.2,121.6,120.2,117.7,112.9,112.7,112.4,101.3$, 68.4, 67.9, 28.9, 28.7, 22.7; HRMS (ESI) $m / z$ for $\mathrm{C}_{27} \mathrm{H}_{24} \mathrm{NO}_{4} \mathrm{~S}[\mathrm{M}+\mathrm{H}]^{+}$calcd. 458.1426, found, 458.1419.

7-\{6-[3-(Benzothiazol-2-yl)phenoxy]hexyloxy\}-2H-chromen-2-one (6y). White powder; yield 47.8\%; $\mathrm{mp} \mathrm{134-}$ $136{ }^{\circ} \mathrm{C} ;{ }^{1} \mathrm{H}$ NMR $\left(400 \mathrm{MHz}, \mathrm{DMSO}-d_{6}\right) \delta: 8.14(\mathrm{~d}, J 7.9 \mathrm{~Hz}, 1 \mathrm{H}$, Benzothiazole-4-H), $8.07(\mathrm{~d}, J 8.0 \mathrm{~Hz}, 1 \mathrm{H}$, Benzothiazole-7-H), 7.97 (d, J 9.5 Hz, 1H, Coumarin-4-H), 7.59 (ddd, J 21.6, 14.1, 7.7 Hz, 4H, Benzothiazol-2phenyl-6- $\mathrm{H}$, Coumarin-5-H, Benzothiazole-5,6-2H), 7.47 (t, J 7.7 Hz, 2H, Benzothiazol-2-phenyl-2,5-2H), 7.15 (d, J $8.1 \mathrm{~Hz}, 1 \mathrm{H}$, Benzothiazol-2-phenyl-4-H), 6.98 (s, 1H, Coumarin-8-H), 6.94 (d, J $8.6 \mathrm{~Hz}, 1 \mathrm{H}$, Coumarin-6-H), 6.27 (d, J $9.5 \mathrm{~Hz}, 1 \mathrm{H}$, Coumarin-3-H), 4.09 (d, J $\left.5.9 \mathrm{~Hz}, 4 \mathrm{H}, 2 \times \mathrm{CH}_{2}\right), 1.78$ (d, J $\left.5.3 \mathrm{~Hz}, 4 \mathrm{H}, 2 \times \mathrm{CH}_{2}\right), 1.51(\mathrm{~s}, 4 \mathrm{H}, 2 \times$

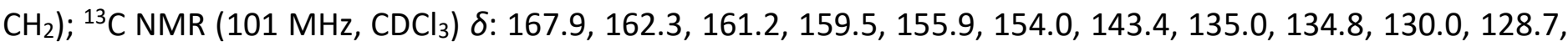
$126.3,125.2,123.2,121.6,120.1,117.6,112.9,112.7,112.4,101.3,68.4,67.9,29.1,28.9,25.8,25.7$; HRMS (ESI) $\mathrm{m} / \mathrm{z}$ for $\mathrm{C}_{28} \mathrm{H}_{26} \mathrm{NO}_{4} \mathrm{~S}[\mathrm{M}+\mathrm{H}]^{+}$calcd. 472.1583, found, 472.1574 .

7-\{2-[3-(Benzothiazol-2-yl)phenoxy]ethoxy\}-4-methyl-2H-chromen-2-one (6z). White powder; yield 65.7\%; mp 189-190 ${ }^{\circ} \mathrm{C} ;{ }^{1} \mathrm{H}$ NMR (400 MHz, DMSO-d $\left.)\right) \delta: 8.17$ (dd, J 7.5, 4.5 Hz, 1H, Benzothiazole-4-H), 8.09 (t, J 7.2 Hz, $1 \mathrm{H}$, Benzothiazole-7-H), 7.85-7.61 (m, 3H, Coumarin-5-H, Benzothiazol-2-phenyl-6-H, Benzothiazole-5-H), 7.62-7.43 (m, 3H, Benzothiazole-6-H, Benzothiazol-2-phenyl-2,5-2H), 7.33-7.15 (m, 1H, Benzothiazol-2phenyl-4-H), 7.10 (d, J $2.4 \mathrm{~Hz}, 1 \mathrm{H}$, Coumarin-8-H), 7.07-7.02 (m, 1H, Coumarin-6-H), 6.23 (s, 1H, Coumarin-3-H), 4.59-4.41 (m, 4H, $\left.2 \times \mathrm{CH}_{2}\right), 2.41\left(\mathrm{~s}, 3 \mathrm{H}, \mathrm{CH}_{3}\right) ;{ }^{13} \mathrm{C} \mathrm{NMR}\left(101 \mathrm{MHz}, \mathrm{CDCl}_{3}\right) \delta: 167.6,161.5,161.2,158.9,155.2$, 
154.0, 152.5, 135.0, 135.0, 130.2, 126.4, 125.6, 125.3, 123.2, 121.6, 120.8, 118.0, 112.8, 112.7, 112.2, 101.6, 67.0, 66.5, 18.6; HRMS (ESI) $\mathrm{m} / z$ for $\mathrm{C}_{25} \mathrm{H}_{20} \mathrm{NO}_{4} \mathrm{~S}[\mathrm{M}+\mathrm{H}]^{+}$calcd. 430.1113, found, 430.1107 .

7-\{3-[3-(Benzothiazol-2-yl)phenoxy]propoxy\}-4-methyl-2H-chromen-2-one (6aa).White powder; yield 53.5\%; mp 117-119 ${ }^{\circ} \mathrm{C} ;{ }^{1} \mathrm{H}$ NMR (400 MHz, DMSO- $\left.d_{6}\right) \delta: 8.15(\mathrm{~d}, J 8.0 \mathrm{~Hz}, 1 \mathrm{H}$, Benzothiazole-4-H), $8.08(\mathrm{~d}, J 8.1 \mathrm{~Hz}, 1 \mathrm{H}$, Benzothiazole-7-H), 7.72-7.60 (m, 3H, Coumarin-5-H, Benzothiazol-2-phenyl-6-H, Benzothiazole-5-H), $7.56(t, J$ $7.7 \mathrm{~Hz}, 1 \mathrm{H}$, Benzothiazole-6-H), 7.52-7.43 (m, 2H, Benzothiazol-2-phenyl-2,5-2H), $7.20(\mathrm{~d}, J 9.0 \mathrm{~Hz}, 1 \mathrm{H}$, Benzothiazol-2-phenyl-4-H), 7.03 (dd, J 9.9, $5.2 \mathrm{~Hz}, 2 \mathrm{H}$, Coumarin-6,8-2H), $6.21(\mathrm{~s}, 1 \mathrm{H}$, Coumarin-3-H), 4.29 (dd, J 13.6, $\left.6.5 \mathrm{~Hz}, 4 \mathrm{H}, 2 \times \mathrm{CH}_{2}\right), 2.39\left(\mathrm{~s}, 3 \mathrm{H}, \mathrm{CH}_{3}\right), 2.31-2.21\left(\mathrm{~m}, 2 \mathrm{H}, \mathrm{CH}_{2}\right) ;{ }^{13} \mathrm{C} \mathrm{NMR}\left(101 \mathrm{MHz}, \mathrm{CDCl}_{3}\right) \delta: 167.8,161.8$, $161.2,159.2,155.2,153.9,152.5,135.0,134.9,130.1,126.3,125.5,125.3,123.2,121.6,120.4,117.8,113.6$, 112.6, 112.5, 112.0, 101.5, 64.9, 64.3, 29.1, 18.6; HRMS (ESI) $m / z$ for $\mathrm{C}_{26} \mathrm{H}_{22} \mathrm{NO}_{4} \mathrm{~S}[\mathrm{M}+\mathrm{H}]^{+}$calcd. 444.1270, found, 444.1263 .

7-\{4-[3-(Benzothiazol-2-yl)phenoxy]butoxy\}-4-methyl-2H-chromen-2-one (6bb).White powder; yield 78.3\%; mp 142-144 ${ }^{\circ} \mathrm{C} ;{ }^{1} \mathrm{H}$ NMR (400 MHz, DMSO- $\left.d_{6}\right) \delta: 8.14(\mathrm{t}, J 6.4 \mathrm{~Hz}, 1 \mathrm{H}$, Benzothiazole-4-H), $8.07(\mathrm{~d}, J 8.0 \mathrm{~Hz}, 1 \mathrm{H}$, Benzothiazole-7-H), 7.71-7.61 (m, 2H, Coumarin-5-H, Benzothiazol-2-phenyl-6-H), 7.57 (dd, J 15.3, 7.2 Hz, 2H, Benzothiazole-5,6-2H), 7.48 (td, J 7.5, 3.8 Hz, 2H, Benzothiazol-2-phenyl-2,5-2H), 7.17 (dd, J 8.2, 1.6 Hz, 1H, Benzothiazol-2-phenyl-4-H), 6.98 (dd, J 12.8, $4.1 \mathrm{~Hz}, 2 \mathrm{H}$, Coumarin-6,8-2H), 6.19 (s, $1 \mathrm{H}$, Coumarin-3-H), 4.19 (s, $\left.4 \mathrm{H}, 2 \times \mathrm{CH}_{2}\right), 2.38\left(\mathrm{~s}, 3 \mathrm{H}, \mathrm{CH}_{3}\right), 1.95\left(\mathrm{~s}, 4 \mathrm{H}, 2 \times \mathrm{CH}_{2}\right) ;{ }^{13} \mathrm{C} \mathrm{NMR}\left(101 \mathrm{MHz}, \mathrm{CDCl}_{3}\right) \delta: 167.86,162.0,161.3,159.3$, $155.2,154.0,152.5,135.0,134.8,130.0,126.3,125.5,125.2,123.2,121.6,120.2,117.6,113.5,112.7,112.5$, 111.8, 101.3, 68.0, 67.6, 25.8, 18.6; HRMS (ESI) $\mathrm{m} / z$ for $\mathrm{C}_{27} \mathrm{H}_{24} \mathrm{NO}_{4} \mathrm{~S}[\mathrm{M}+\mathrm{H}]^{+}$calcd. 458.1426, found, 458.1417.

7-\{5-[3-(Benzothiazol-2-yl)phenoxy]pentyloxy\}-4-methyl-2H-chromen-2-one (6cc). Yellow powder; yield 44.6\%; mp 114-116 ${ }^{\circ} \mathrm{C}$; ${ }^{1} \mathrm{H}$ NMR (400 MHz, DMSO-d $) \delta: 8.14$ (d, J $7.8 \mathrm{~Hz}, 1 \mathrm{H}$, Benzothiazole-4-H), 8.06 (d, J 8.0 $\mathrm{Hz}, 1 \mathrm{H}$, Benzothiazole-7-H), 7.66-7.52 (m, 4H, Coumarin-5-H, Benzothiazol-2-phenyl-6-H, Benzothiazole-5,62H), 7.47 (t, J $7.8 \mathrm{~Hz}, 2 \mathrm{H}$, Benzothiazol-2-phenyl-2,5-2H), 7.15 (dd, J 8.2, 2.1 Hz, 1H, Benzothiazol-2-phenyl-4-H), 6.98-6.91 (m, 2H, Coumarin-6,8-2H), 6.19 (t, J $2.9 \mathrm{~Hz}, 1 \mathrm{H}$, Coumarin-3-H), $4.11\left(\mathrm{t}, \mathrm{J} 5.8 \mathrm{~Hz}, 4 \mathrm{H}, 2 \times \mathrm{CH}_{2}\right), 2.37$ (s, $\left.3 \mathrm{H}, \mathrm{CH}_{3}\right), 1.89-1.78\left(\mathrm{~m}, 4 \mathrm{H}, 2 \times \mathrm{CH}_{2}\right), 1.66-1.50\left(\mathrm{~m}, 2 \mathrm{H}, \mathrm{CH}_{2}\right) ;{ }^{13} \mathrm{C} \mathrm{NMR}\left(101 \mathrm{MHz}, \mathrm{CDCl}_{3}\right) \delta: 167.9,162.1,161.3$, $159.5,155.2,153.9,152.5,135.0,134.8,130.0,126.3,125.5,125.2,123.2,121.6,120.1,117.7,113.4,112.7$, 112.6, 111.8, 101.3, 68.3, 67.9, 28.9, 28.7, 22.7, 18.6; HRMS (ESI) $m / z$ for $\mathrm{C}_{28} \mathrm{H}_{26} \mathrm{NO}_{4} \mathrm{~S}[\mathrm{M}+\mathrm{H}]^{+}$calcd. 472.1583, found, 472.1573 .

7-\{6-[3-(Benzothiazol-2-yl)phenoxy]hexyloxy\}-4-methyl-2H-chromen-2-one (6dd). White powder; yield 56.6\%; $\mathrm{mp} 128-130{ }^{\circ} \mathrm{C} ;{ }^{1} \mathrm{H}$ NMR (400 MHz, DMSO- $\left.d_{6}\right) \delta: 8.15(\mathrm{~d}, J 7.8 \mathrm{~Hz}, 1 \mathrm{H}$, Benzothiazole-4-H), $8.07(\mathrm{~d}, J 8.1 \mathrm{~Hz}, 1 \mathrm{H}$, Benzothiazole-7-H), 7.70-7.59 (m, 3H, 4H, Coumarin-5-H, Benzothiazol-2-phenyl-6- $\mathrm{H}$, Benzothiazole-5- $\mathrm{H}), 7.56$ (t, J $7.6 \mathrm{~Hz}, 1 \mathrm{H}$, Benzothiazole-6-H), 7.48 (t, J $7.6 \mathrm{~Hz}, 2 \mathrm{H}$, Benzothiazol-2-phenyl-2,5-2H), $7.16(\mathrm{~d}, J 8.1 \mathrm{~Hz}, 1 \mathrm{H}$, Benzothiazol-2-phenyl-4-H), $6.96(\mathrm{~d}, J 10.3 \mathrm{~Hz}, 2 \mathrm{H}$, Coumarin-6,8-2H), $6.20(\mathrm{~s}, 1 \mathrm{H}$, Coumarin-3-H), $4.10(\mathrm{t}, J 5.8$ $\left.\mathrm{Hz}, 4 \mathrm{H}, 2 \times \mathrm{CH}_{2}\right), 2.38\left(\mathrm{~s}, 3 \mathrm{H}, \mathrm{CH}_{3}\right), 1.79\left(\mathrm{~d}, J 4.9 \mathrm{~Hz}, 4 \mathrm{H}, 2 \times \mathrm{CH}_{2}\right), 1.52\left(\mathrm{~s}, 4 \mathrm{H}, 2 \times \mathrm{CH}_{2}\right) ;{ }^{13} \mathrm{C} \mathrm{NMR}\left(101 \mathrm{MHz} \mathrm{CDCl}_{3}\right)$ $\delta: 167.9,162.1,161.3,159.5,155.2,154.0,152.6,135.0,134.8,130.0,126.3,125.4,125.2,123.2,121.6,120.0$, 117.6, 113.4, 112.7, 112.6, 111.7, 101.3, 68.4, 67.9, 29.1, 28.9, 25.8, 25.7, 18.6; HRMS (ESI) $m / z$ for $\mathrm{C}_{29} \mathrm{H}_{28} \mathrm{NO}_{4} \mathrm{~S}$ $[\mathrm{M}+\mathrm{H}]^{+}$calcd. 486.1739 , found, 486.1735 .

Crystallographic study. The single crystal of $6 \mathbf{v}$ was obtained from the mixed liquor of chloroform and methanol ( $v / v=1: 1)$. The $X$-ray single crystal diffraction data were collected on an Agilent SuperNova (Dual, $\mathrm{Cu}$ at zero, AtlasS2) diffractometer at $100.00(10) \mathrm{K}$ using Mo K $\alpha$ radiation $(\lambda=0.71073 \AA$ ) by the $\omega$ scan mode. The program CrysAlisPro was used for integration of the diffraction profiles. ${ }^{22}$ The structure was solved by direct methods using the SHELXS program of the SHELXTL package and refined by full-matrix least-squares methods with SHELXL. ${ }^{23}$ All non-hydrogen atoms of the compound $6 \mathbf{v}$ were refined with anisotropic thermal parameters. All hydrogen atoms were observed and placed at their calculated positions with a fixed value of their isotropic displacement parameters. 
In vivo insecticidal activity assay. The test insects Plutella xylostella and Aphis fabae were reared at $25( \pm 1){ }^{\circ} \mathrm{C}$ and $60( \pm 5) \%$ relative humidity in a greenhouse. The bioactivity of title compounds against $P$. xylostella and aphid ( $A$. fabae) were tested according to the modified leaf-dip method using the reported procedure. ${ }^{24,25}$ The compounds were dissolved in DMF and then diluted with water (containing $0.1 \%$ Triton X-100). The disks of Chinese cabbage leaves were prepared and dipped in a test solution for $10 \mathrm{~s}$. After drying, the leaves were placed in a plastic dish with ten third instar larvae ( $P$. xylostella). The mortality was determined after $120 \mathrm{~h}$ by the dead insect number in the treated dishes relative to that in the untreated controls. At the same time, Horsebean seedlings with apterous adult $A$. fabae were prepared and dipped in a test solution for $10 \mathrm{~s}$. After drying, the seedlings were placed in a glass bottle. The mortality was determined after $72 \mathrm{~h}$. All the treatments and controls were carried out in three replicates.

\section{Acknowledgements}

This work was supported by the Fundamental Research Funds for the Central Universities of China (No. KYTZ201604 and KJSY201607).

\section{Supplementary Material}

Data of the key intermediates, copies of ${ }^{1} \mathrm{H}$ and ${ }^{13} \mathrm{C}$ NMR spectra of the new compounds, and crystallographic data of compound $\mathbf{6 v}$.

\section{References}

1. Wang, Y.B. Pesticide 2003, 41, 10-12.

http://doi.org/10.16820/j.crki.1006-0413.2002.11.003

2. Torres-Vila, L.M.; Rodrıguez-Molin, M.C.; Lacasa-Plasencia, A.; Bielza-Lino, P. Crop Protection 2002, 21, 1003-1013.

https://doi.org/10.1016/S0261-2194(02)00081-9

3. Aktar M.W.; Sengupta, D.; Chowdhury, A. Interdis. Toxicol. 2009, 2, 1-12.

https://doi.org/10.2478/v10102-009-0001-7

4. Havrylyuk, D.; Mosula, L.; Zimenkovsky, B.; Vasylenko, O.; Gzella, A.; Lesyk, R. Eur. J. Med. Chem. 2010, 45, 5012-5021.

https://doi.org/10.1016/j.ejmech.2010.08.008

5. Praveen, C.; Nandakumar, A.; Dheenkumar, P.; Muralidharan, D.; Perumal, P.T.; J. Chem. Sci. 2012, 124, 609-624.

https://doi.org/10.1007/s12039-012-0251-3

6. Shafi, S.; Alam, M.M.; Mulakayala, N.; Mulakayala, C.; Vanaja, G.; Kalle, A.M.; Pallu, R.; Alam, M.S. Eur. J. Med. Chem. 2012, 49, 324-333.

https://doi.org/10.1016/j.ejmech.2012.01.032

7. Telvekar, V.N.; Bairwa, V.K.; Satardekar, K.; Bellubi A. Bioorg. Med. Chem. Lett. 2012, 22, 649-652. https://doi.org/10.1016/j.bmcl.2011.10.064 
8. Bondock, S.; Fadaly, W.; Metwally, M.A. Eur. J. Med. Chem. 2010, 45, 3692-3701.

https://doi.org/10.1016/j.ejmech.2010.05.018

9. Liu, J.Z.; Song, B.A.; Bhadury, P.S.; Hu, D.Y.; Yang, S. Phosphorus, Sulfur, and Silicon. 2012, 187, 61-70. https://doi.org/10.1080/10426507.2011.575422

10. Li, J.; Wang, Z.Y.; Wu, Q.Y.; Yang, G.F. Pest Manag. Sci. 2015, 71, 694-700. https://doi.org/10.1002/ps.3827

11. Wang, Y.Z. M.A. Thesis, Central China normal university, Wuhan, China 2008.

12. Hubbard, W.L.; Grahame, R.E.; Covey, R.A., Jancis, E.H. U.S. Patent 4020 165, 1977.

13. Sandhu, S.; Bansal, Y.; Silakari, O.; Bansal, G. Bioorg. Med. Chem. Lett. 2014, 22, 3806-3814.

http://dx.doi.org/10.1016/j.bmc.2014.05.032

14. Nasr, T.; Bondock, S.; Youns, M. Eur. J. Med. Chem. 2014, 76, 539-548.

http://dx.doi.org/10.1016/j.ejmech.2014.02.026

15. Pingaew, R.; Saekee, A.; Mandi, P.; Nantasenamat, C.; Prachayasittikul, S.; Ruchirawat, S.; Prachayasittikul, V. Eur. J. Med. Chem. 2014, 85, 65-76.

http://dx.doi.org/10.1016/j.ejmech.2014.07.087

16. Fylaktakidou, K.C.; Hadjipavlou-Litina, D. J.; Litinas, K. E.; Nicolaides, D. N. Curr. Pharm. Design. 2004, 10, 3813-3833.

\section{https://doi.org/10.2174/1381612043382710}

17. Renuka, N.; Kumar, K.A. Bioorg. Med. Chem. Lett. 2013, 23, 6406-6409.

http://dx.doi.org/10.1016/j.bmcl.2013.09.053

18. Khan, K.M.; Saify, Z.S.; Hayat, S.; Khan, M.Z.; Noor, F.; Makhmoor, T.; Choudhary, M.I.; Perveen, S. J. Chem. Soc. Pak. 2002, 24, 226-231.

19. Liu, C.L.; Li, M.; Guan, A.Y.; Zhang, H.; Li, Z.N., Ye, T.J. CN Patent 1869032A, 2006.

20. Xie, S.S.; Wang, X.B.; Li, J.Y.; Yang, L.; Kong, L.Y. Eur. J. Med. Chem. 2013, 64, 540-553.

http://dx.doi.org/10.1016/j.ejmech.2013.03.051

21. Khan, K.M.; Rahim, F.; Halim, S.A.; Taha, M.; Khan, M.; Perveen, S.; Mesaik, M.A.; Choudhary, M.I. Bioorg. Med. Chem. Lett. 2011, 19, 4286-4294.

https://doi.org/10.1016/j.bmc.2011.05.052

22. Agilent C.P. CrysAlis PRO. Agilent Technologies, Yarnton, Oxfordshire, England 2013.

23. Sheldrick, G.M. Acta Crystallogr. A. 2008, 64, 112-122.

https://doi.org/10.1107/S0108767307043930

24. Wu, C.C.; Wang, B.L.; Liu, J.B.; Wei, W.; Li, Y.X.; Liu, Y.; Chen, M.G.; Xiong, L.X.; Yang, N.; Li, Z.M. Chin. Chem. Lett. 2017, 28, 1248-1251.

http://dx.doi.org/10.1016/j.cclet.2017.01.019

25. Zhao, J.H.; Ji, M. H.; Xu, X.H.; Cheng J.L.; Zhu, G.N.; Chin. Chem. Lett. 2010, 21, 1391-1394. https://doi.org/10.1016/j.cclet.2010.06.035 